Silvana de Barros

\title{
Efeito da respiração lenta na pressão arterial e na função autonômica em hipertensos
}

Tese apresentada à Faculdade de Medicina da Universidade de São Paulo para obtenção do título de Doutora em Ciências

Programa de Nefrologia

Orientador: Prof. Dr. Décio Mion Junior

São Paulo

2017 
Dados Internacionais de Catalogação na Publicação (CIP)

Preparada pela Biblioteca da

Faculdade de Medicina da Universidade de São Paulo

Creprodução autorizada pelo autor

Barros, Silvana de

Efeito da respiração lenta na pressão arterial e na função autonômica em hipertensos / Silvana de Barros -- São Paulo, 2017.

Tese(doutorado)--Faculdade de Medicina da Universidade de São Paulo. Programa de Nefrologia.

Orientador: Décio Mion Junior.

Descritores: 1.Respiração lenta guiada 2.Hipertensão 3.Tratamento não farmacológico 4.Sistema nervoso simpático 5.Catecolaminas/sangue

USP/FM/DBD-205/17 


\section{Dedicatória}

À minha querida mãe, Pedrina, pelo amor incondicional, e por ter me ensinado, da maneira mais carinhosa possível, a ser forte e a enfrentar os embates da vida.

Ao meu irmão-pai, Jessé, pelo incentivo desde a mais tenra idade e por todo carinho dedicado.

Ao meu amado esposo, Giovanio, pelo incentivo, apoio, amizade, companheirismo, paciência e principalmente amor constante.

Ao meu pequeno e amado filho, Nicolas, meu melhor e maior projeto de vida. Responsável pelas alegrias mais simples (porém inesquecíveis) e doces de toda minha vida. 


\section{Agradecimentos}

Agradeço a todos que colaboraram de forma direta ou indireta para realização deste trabalho e, em especial:

Ao Prof. Dr. Décio Mion Junior pela oportunidade de aprendizado com o profissional competente e formador acadêmico que representa. Igualmente pela confiança depositada e principalmente por ter me instigado a sempre procurar novos caminhos e respostas na busca do conhecimento.

Ao Dr. Giovanio Vieira da Silva, e neste momento me refiro a ele como profissional, pelo auxílio constante, paciência e conhecimento compartilhado.

À amiga, Dra Josiane Lima de Gusmão, pelos risos intermináveis, por me ouvir nos momentos mais críticos, aconselhar, dividir saberes, e principalmente por sofrer e se alegrar comigo nos inúmeros e cansativos exames de microneurografia.

Ao Dr. Crivaldo Gomes Cardoso Junior, Dra Kátia Coelho Ortega e Dra Bruna Oneda pelo conhecimento partilhado e importantes sugestões na fase de construção deste projeto.

À Tatiana Goveia de Araújo e Dinoélia Rosa de Souza, mais que companheiras de pós-graduação, por todo auxílio nos momentos necessários.

As secretárias do Laboratório de Hipertensão, Elisa e Cristina, por estarem dispostas a ajudar sempre que solicitado.

Aos meus familiares, que sempre torceram por mim e estiveram ao meu lado em todos os momentos.

Aos voluntários que participaram deste estudo. Sem vocês, sua ajuda e paciência, nada seria possível. 
"Existem muitas hipóteses em ciência que estão erradas. Isso é perfeitamente aceitável, elas são a abertura para achar as que estão certas".

Carl Sagan 


\section{Normalização Adotada}

Esta tese está de acordo com:

Referências: adaptado de International Comittee of Medical Journals Editors (Vancouver)

Universidade de São Paulo. Faculdade de Medicina. Divisão de Biblioteca e Documentação. Guia de apresentação de dissertações, teses e monografias. Elaborado por Anneliese Carneiro da Cunha, Maria Julia de A. L. Freddi, Maria F. Crestana, Marinalva de Souza Aragão, Suely Campos Cardoso, Valéria Vilhena. 3a ed. São Paulo: Divisão de Biblioteca e Documentação; 2011.

Abreviaturas dos títulos dos periódicos de acordo com List of Journals indexed in Index Medicus. 


\section{SUMÁRIO}

\section{LISTA DE ABREVIATURAS}

LISTA DE FIGURAS

LISTA DE TABELAS

RESUMO

ABSTRACT

1. INTRODUÇÃO 1

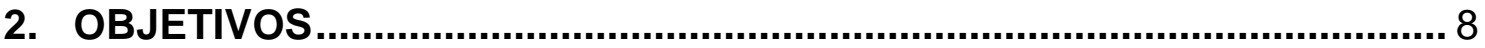

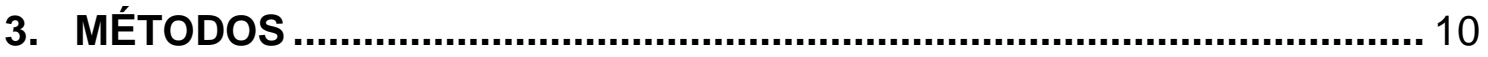

3.1- Desenho do Estudo ............................................................... 11

3.2- Casuística ............................................................................... 11

3.2.1 - CRITÉRIOS DE INCLUSÃO ................................................. 11

3.2.2- CRITÉRIOS DE EXCLUSÃO .................................................... 12

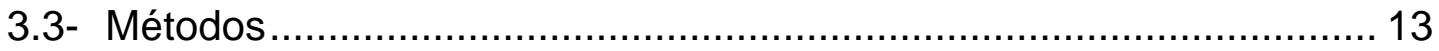

3.3.1 - PROTOCOLO GERAL DO ESTUdO............................................. 13

3.3.2- MedidAS dA Pressão ARTERIAL ........................................... 16

3.3.2.1 Monitorização Ambulatorial da Pressão Arterial (MAPA).. 16

3.3.2.2 Pressão arterial de consultório .................................. 17

3.3.3- FREQUÊNCIA CARDÍACA ..................................................... 17

3.3.4- DOSAGENS DE CATECOLAMINAS PLASMÁTICAS ............................ 18

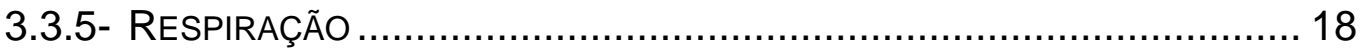

3.3.6- ATIVIDADE NERVOSA SIMPÁTICA PERIFÉRICA .............................. 19

3.3.7- CONTROLE NEUROVASCULAR................................................ 20

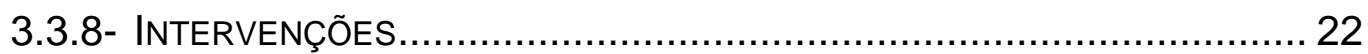

3.3.9- AdESÃO À INTERVENÇÃO .............................................. 23

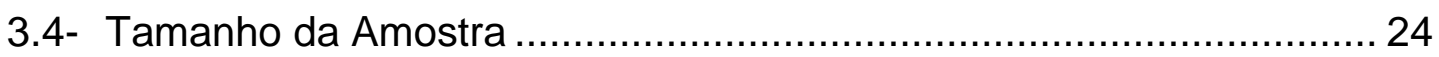

3.5- Análise estatística................................................................ 25 
4. RESULTADOS

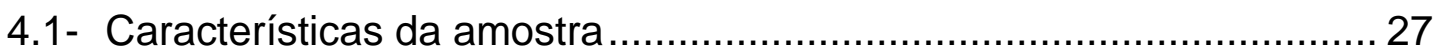

4.2- Pressão arterial de consultório, MAPA de 24 horas e frequência

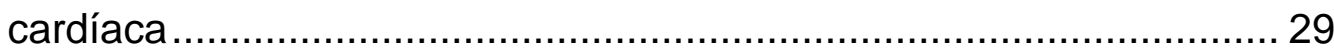

4.3- Atividade nervosa simpática - concentração de noradrenalina plasmática

4.4- Características da amostra - subgrupo dos participantes com microneurografia

4.5- Pressão arterial de consultório, MAPA de 24 horas e frequência cardíaca - subgrupo dos participantes com microneurografia

4.6- Atividade nervosa simpática - Concentração de noradrenalina plasmática e microneurografia.

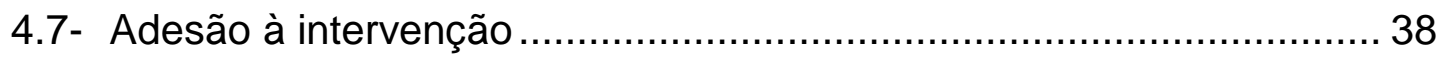

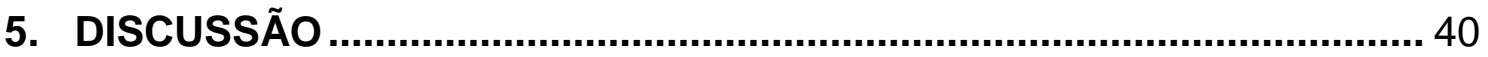

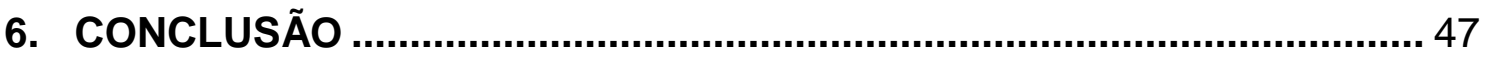

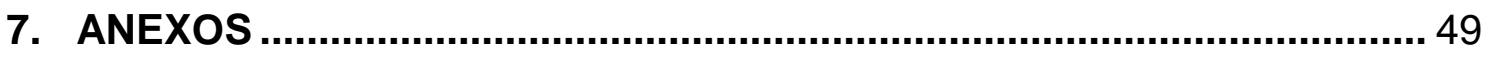

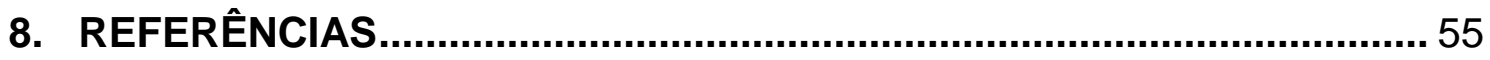




\section{Lista de Abreviaturas}

ANS Atividade Nervosa Simpática

ANSP Atividade Nervosa Simpática Periférica

AVC Acidente Vascular Cerebral

DCV Doença Cardiovascular

DM2 Diabetes Mellitus Tipo 2

EDTA Ácido Etilenodiamino Tetra-Acético

FC

Frequência Cardíaca

FR

Frequência Respiratória

GC Grupo Controle

GRL Grupo Respiração Lenta

HA Hipertensão Arterial

HC-FMUSP Hospital das Clínicas da Faculdade de Medicina da Universidade de São Paulo

HPLC-ED High-Performance Liquid Chromatography with Electrochemical Detection

MAPA Monitorização Ambulatorial da Pressão Arterial

MDRD Modification of Diet in Renal Disease

MRPA Monitorização Residencial da Pressão Arterial 
PA Pressão Arterial

PAD Pressão Arterial Diastólica

PAM Pressão Arterial Média

PAS Pressão Arterial Sistólica

TCLE Termo de Consentimento Livre e Esclarecido 


\section{Lista de Figuras}

Figura 1 - Esquematização geral do estudo

Figura 2 - Técnica de microneurografia durante sessão experimental: posicionamento do eletrodo ativo e referência na perna esquerda de um participante

Figura 3 - Estímulo físico isométrico durante sessão experimental: participante realizando a preensão manual com o dinamômetro .. 21

Figura 4 - RESPeRATE ${ }^{\circledR}$ (Intercure, Lod, Israel)

Figura 5 - Fluxograma de entrada e saída dos participantes durante o estudo

Figura 6 - Concentração de noradrenalina pré e pós intervenção, nos grupos controle e respiração lenta

Figura 7 - Concentração de noradrenalina pré e pós intervenção, nos grupos controle e respiração lenta; subgrupo dos participantes com microneurografia

Figura 8 - Atividade nervosa simpática periférica (ANSP) medida pela microneurografia antes e após intervenção no Grupo Respiração Lenta (GRL) e Grupo Controle (GC) 


\section{Lista de Tabelas}

Tabela 1 - Características demográficas, clínicas e laboratoriais dos grupos respiração Lenta (GRL) e controle (GC)

Tabela 2 - Comportamento da pressão arterial de consultório e MAPA de 24 horas antes e após a intervenção, de acordo com a randomização. Grupo Respiração Lenta (GRL) versus Grupo Controle (GC)

Tabela 3 - Pressão arterial (Consultório e MAPA de 24 horas) e concentração de noradrenalina plasmática, antes e após a intervenção, de acordo com a randomização e atividade nervosa simpática basal (mediana da noradrenalina plasmática). Grupo Respiração Lenta (GRL) versus Grupo Controle (GC)

Tabela 4 - Características demográficas, clínicas e laboratoriais dos grupos controle $(G C)$ e respiração lenta $(G R L)$; subgrupo dos voluntários com microneurografia

Tabela 5 - Comportamento da pressão arterial de consultório e pela MAPA de 24 horas antes e após a intervenção de acordo com a randomização, Grupo Respiração Lenta (GRL) versus Grupo Controle (GC); subgrupo dos voluntários com microneurografia... 35

Tabela 6 - Atividade Nervosa Simpática Periférica (ANSP), medida pela microneurografia, antes e após a intervenção de acordo com a randomização, Grupo Respiração Lenta (GRL) versus Grupo Controle (GC)

Tabela 7 - Adesão ao exercício de respiração lenta guiada 


\section{Resumo}

Barros S. Efeito da respiração lenta na pressão arterial e na função autonômica em hipertensos [tese]. São Paulo: Faculdade de Medicina, Universidade de São Paulo; 2017.

INTRODUÇÃO: A respiração lenta é indicada como tratamento não medicamentoso da hipertensão arterial. Porém, os mecanismos fisiológicos envolvidos na redução da pressão arterial (PA) ainda são desconhecidos. A redução na atividade nervosa simpática (ANS) pode ser um dos mecanismos envolvidos na redução da PA. OBJETIVOS: Avaliar o efeito crônico da respiração lenta na PA e na ANS em hipertensos. MÉTODOS: Foram randomizados hipertensos, com e sem uso de anti-hipertensivos, em grupo controle (GC), orientados a ouvir músicas serenas com uso de aparelho de MP3, ou grupo respiração lenta $(G R L)$, treinados a reduzir a frequência respiratória com auxílio de um dispositivo eletrônico, tendo como alvo terapêutico uma frequência respiratória menor que 10 respirações por minuto, por um período de 15 minutos diários, durante 8 semanas. Antes e após 0 período de intervenção, foi realizada monitorização ambulatorial da pressão arterial (MAPA), dosagem de catecolaminas plasmáticas e medida da atividade nervosa simpática periférica (ANSP) pela técnica da microneurografia. RESULTADOS: Completaram o estudo 17 voluntários no GRL e 15 no GC. Não houve mudança na PA de consultório antes e após a intervenção nos dois grupos. Observou-se redução na pressão arterial sistólica (PAS) e diastólica (PAD) na vigília entre os períodos pré e pós-intervenção apenas no GC $(131 \pm 10 / 92 \pm 9$ vs $128 \pm 10 / 88 \pm 8 \mathrm{mmHg}, \mathrm{p}<0,05)$. Não foi observada diferença na concentração de catecolaminas plasmáticas $(\mathrm{pg} / \mathrm{ml})$ em ambos os grupos entre os períodos pré e pós-intervenção: GRL 302 (220-256) vs 234 (156-318), $p=0,35$; e GC 201 (144-230) vs 221 (179-274), $p=0,97$. Nos voluntários que realizaram microneurografia, $\mathrm{GRL}(\mathrm{n}=10)$ e $\mathrm{GC} \quad(n=10)$, observou-se redução significativa da PAD de sono entre os períodos pré e pósintervenção apenas no GC $(83 \pm 6$ vs $79 \pm 4 \mathrm{mmHg}, p<0,05)$ A ANSP (impulsos/minuto) medida pela microneurografia apresentou elevação no período pós-intervenção em comparação ao período pré-intervenção nos dois grupos: GRL $(16 \pm 6$ vs $22 \pm 8, p<0,05)$ e $G C(20 \pm 5$ vs $23 \pm 5, p<0,05)$. CONCLUSÕES: A respiração lenta, realizada por 15 minutos diários durante 8 semanas, não reduziu a pressão arterial, os níveis de catecolaminas plasmáticas e a atividade nervosa simpática periférica de hipertensos.

Descritores: Respiração lenta guiada; hipertensão; tratamento não farmacológico; sistema nervoso simpático; microneurografia; catecolaminas/sangue. 


\begin{abstract}
Barros S. Effect of slow breathing on blood pressure and autonomic function in hypertensive patients [thesis]. São Paulo: "Faculdade de Medicina, Universidade de São Paulo"; 2017.

INTRODUCTION: Slow breathing is indicated as nonpharmacological treatment of hypertension. However, the physiological mechanisms involved in blood pressure (BP) reduction are still unknown. The decrease in sympathetic nerve activity (SNA) may be one of the mechanisms involved in BP reduction. OBJECTIVES: To evaluate the chronic effect of slow breathing on BP and SNS in hypertensive patients. METHODS: Hypertensive patients, with or without use of antihypertensive drugs, were randomized to listen serene songs using an MP3 player (Control Group - CG) or device-guided slow breathing group (DGB), who were trained to reduce respiratory rate with assistance of an electronic device, targeting a respiratory rate of less than 10 breaths per minute, for a period of 15 minutes per day for 8 weeks. Before and after the intervention period, ambulatory blood pressure monitoring (ABPM), plasma catecholamines concentration and muscle sympathetic nerve activity (MSNA) using the microneurography technique were performed. RESULTS: 17 volunteers in the DGB and 15 in the CG completed the study. There was no change in office BP before and after intervention in both groups. There was a reduction in daytime systolic (SBP) and diastolic (DBP) before and after intervention only in the CG $(131 \pm 10 / 92 \pm 9$ vs $128 \pm 10 / 88 \pm 8 \mathrm{mmHg}, \mathrm{p}<0,05)$. No difference in plasma catecholamines concentration $(\mathrm{pg} / \mathrm{ml})$ was observed in both groups before and after intervention: DGB 302 (220-256) vs 234 (156-318), p = 0.35; CG 201 (144-230) vs 221 (179-274), $p=0.97$. In the volunteers who underwent microneurography, DGB $(n=10)$ and CG $(n=10)$, there was a significant reduction in sleep DBP only in the CG: $83 \pm 6$ vs $79 \pm 4 \mathrm{mmHg}, p<0,05$. The MSNA (bursts/minute) measured by the microneurography showed a rise after the intervention in both groups: DGB $(16 \pm 6$ vs $22 \pm 8, p<0.05)$ and CG $(20 \pm 5$ vs $23 \pm 5, p<0.05)$. CONCLUSIONS: Slow breathing, performed for 15 minutes daily for 8 weeks, did not reduce blood pressure, plasma catecholamine concentration and muscle sympathetic nerve activity in hypertensive patients.
\end{abstract}

Descriptors: Device-guided slow breathing; hypertension; nonpharmacological treatment; sympathetic nervous system; microneurography; catecholamines/bood. 


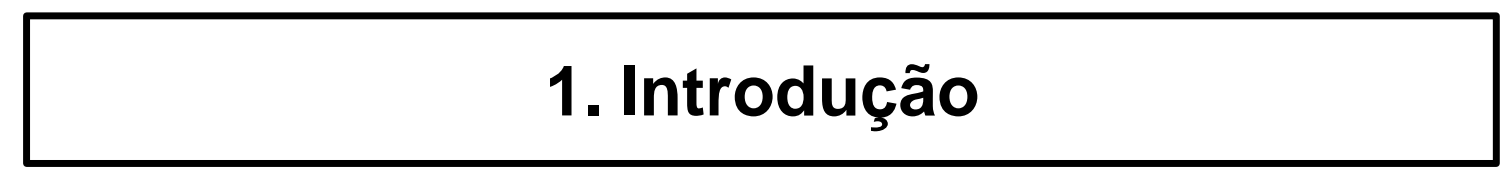


A hipertensão arterial (HA) é a doença crônica com maior prevalência em países desenvolvidos e em desenvolvimento ${ }^{1}$, representando um importante fator de risco para doenças cardiovasculares (DCV $)^{2}$. Estudo transversal realizado em comunidades rurais e urbanas de países de alta, média e baixa renda ${ }^{1}$ mostrou que a $\mathrm{HA}$ acomete em torno de $40 \%$ da população mundial e apresenta tendência de elevação com o avançar da idade. No Brasil essa prevalência é de aproximadamente $30 \%$, de acordo com estudos populacionais realizados em várias cidades ${ }^{3}$.

A mortalidade por DCV é a principal causa de óbito em nosso meio, responsável por 29,8\% das mortes na população brasileira ${ }^{2}$. No ano de 2015 , foram registradas 808.672 internações por DCV, tais como doenças isquêmicas do coração, doenças cerebrovasculares, insuficiência cardíaca e outras complicações da $\mathrm{HA}^{4}$.

Visto a HA ser o principal fator de risco para DCV, o seu tratamento é parte fundamental nas estratégias que visam diminuir a morbi-mortalidade cardiovascular da população. Uma meta-análise ${ }^{5}$ com 147 estudos randomizados mostrou que reduções de $10 \mathrm{mmHg}$ na pressão arterial sistólica (PAS) ou de $5 \mathrm{mmHg}$ na pressão arterial diastólica (PAD) poderiam alcançar diminuições em torno de $22 \%$ em todos os eventos coronarianos e em $41 \%$ dos acidentes vasculares cerebrais (AVC).

O tratamento da HA realizado por meio de intervenções farmacológicas proporciona significativa redução nos níveis de pressão arterial ${ }^{6}$, morbidade e mortalidade cardiovascular, porém apresentam efeitos colaterais e custos significativos, fatores estes que ajudam a explicar a baixa adesão ao tratamento prescrito $^{2}$. Portanto, há concordância entre as diversas diretrizes clínicas para o 
manejo da HA sobre a necessidade de associação de tratamentos não farmacológicos efetivos ${ }^{2,7,8}$.

Dentre as terapias não medicamentosas, mudanças no estilo de vida tais como melhora do padrão alimentar por meio de dietas ricas em potássio e

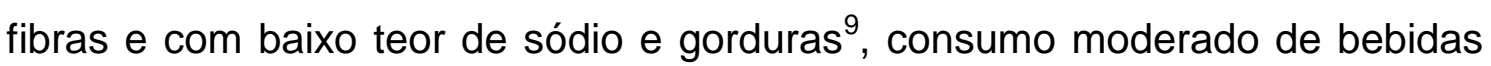
alcoólicas, controle do peso corporal e prática regular de exercícios físicos são indicados como tratamento complementar no controle da $\mathrm{HA}^{2}$.

Além desses recursos, estudos demonstram que técnicas de relaxamento ${ }^{10}$,

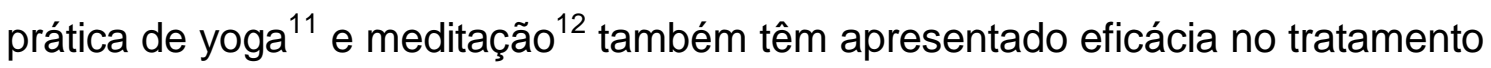
da HA. Embora o mecanismo responsável pela redução da pressão arterial pelo emprego destas técnicas não seja claro, o fato de todas elas levarem ao denominador comum de redução da frequência respiratória, nos faz levantar a hipótese de que esta alteração fisiológica induzida por essas técnicas seja o elo responsável pela redução da pressão arterial observada ${ }^{13}$.

Baseado nesse contexto e com o objetivo de reduzir os valores de pressão arterial (PA), foi desenvolvido um dispositivo eletrônico, (RESPeRATE ${ }^{\circledR}$ ), que interage com o indivíduo por meio de uma melodia composta por dois tons diferentes, um para inspiração e outro para expiração. O usuário sincroniza sua respiração com a melodia e o aparelho, gradualmente, prolongará o tom de expiração, induzindo o indivíduo a respirar mais lentamente.

A eficácia deste aparelho de respiração lenta guiada e sua contribuição na modulação respiratória e na redução da PA foram avaliadas em dois trabalhos que compararam o dispositivo eletrônico a um walkman que reproduzia músicas serenas. Um deles ${ }^{14}$, estudo randomizado e duplo cego, realizado com 65 hipertensos que utilizavam ou não drogas anti-hipertensivas, 
e que fizeram uso do dispositivo eletrônico por 10 minutos diários no decorrer de 8 semanas consecutivas mostrou que a redução da pressão arterial de consultório foi significativamente maior no grupo que utilizou o aparelho de respiração guiada: $-15,2 \mathrm{mmHg}$ na PAS, $-10,0 \mathrm{mmHg}$ na PAD e $-11,7 \mathrm{mmHg}$ na pressão arterial média (PAM), comparado com o grupo que utilizou o walkman com músicas serenas: -11,3 mmHg PAS, -5,6 mmHg PAD e -7,5 mmHg PAM. O segundo estudo ${ }^{15}$ realizado com 33 pacientes com PA não controlada, com uso do dispositivo eletrônico por 10 minutos diários durante 8 semanas mostrou reduções de -7,5/ -4,0 mmHg (PAS/PAD) para o grupo ativo e -2,9/-1,5 mmHg (PAS/PAD) para o grupo controle $(p=0,001 / 0,12)$ respectivamente.

Em outro estudo ${ }^{16}$, controlado e duplo-cego, realizado com 149 hipertensos com uso de terapia medicamentosa, metade dos pacientes recebeu o dispositivo eletrônico e o utilizou durante 15 minutos diários por 8 semanas. Os demais pacientes mantiveram apenas acompanhamento clínico, sendo que todos eles realizaram Monitorização Residencial da Pressão Arterial (MRPA) durante todo o estudo. Foi verificado significativa redução da PAS de consultório no grupo que utilizou o dispositivo de respiração lenta $(-15 \pm 1,8 \mathrm{mmHg})$ comparado com o grupo controle $(-9 \pm 1,6 \mathrm{mmHg} p=0,001)$.

O aparelho de respiração lenta também se mostrou eficaz no tratamento de 66 pacientes hipertensos com Diabetes Mellitus tipo II (DM2), não dependentes de insulina, com ou sem terapia medicamentosa anti-hipertensiva, e que realizaram o tratamento por 15 minutos diários, durante 8 semanas. A PAS e PAD do grupo tratamento foi reduzida, $-10 \pm 2 \mathrm{mmHg}$ e $-4 \pm 1 \mathrm{mmHg}(p<0,0001$ e $\mathrm{p}<0,01)$ respectivamente, mas não no grupo controle, $\mathrm{PAS}+1 \pm 2 \mathrm{mmHg} \mathrm{e}$ PAD $-1 \pm 1 \mathrm{mmHg}, \mathrm{p}>0,05$ para ambos ${ }^{17}$. 
A adesão ao tratamento com o dispositivo de respiração lenta guiada, também foi avaliada em outro estudo ${ }^{18}$, que demonstrou que este foi utilizado pelos pacientes em $74 \%$ dos dias durante o período de 8 semanas, sendo que em $97 \%$ das vezes foram completados os 15 minutos de duração da sessão, o que comprova a boa aceitação dos pacientes na utilização do aparelho.

Contudo, há estudos ${ }^{19,20}$ na literatura, porém em menor número, que demonstram efeitos desfavoráveis à eficácia do dispositivo de respiração lenta guiada no controle da PA. A despeito de haver tais controvérsias nos resultados, o dispositivo eletrônico é recomendado pela American Heart Association, como uma opção de tratamento não medicamentoso eficaz para a redução da PA (recomendação classe IIA, nível de evidência $B)^{21}$.

Entretanto, os mecanismos pelos quais a diminuição da frequência respiratória (FR) reduz a PA ainda não são plenamente conhecidos.

Evidências científicas demonstram que uma FR lenta e profunda, de aproximadamente seis respirações por minuto, leva a um aumento da sensibilidade barorreflexa e uma consequente redução da PA em indivíduos hipertensos $^{13,22,23}$.

Outro mecanismo proposto seria que a modulação da atividade nervosa simpática (ANS) e da resistência vascular periférica poderia ser afetada pela FR. Reduções na FR em torno de 6 a 10 respirações por minuto, ao aumentarem o volume corrente, estimulariam os receptores de estiramento cardiopulmonares, que por sua vez, reduziriam a descarga das fibras simpáticas eferentes, resultando em uma diminuição da resistência vascular sistêmica e consequente redução da $\mathrm{PA}^{24}$. 
No entanto, tais afirmações são apenas hipóteses. Até o momento, não foi categoricamente comprovado que a redução da ANS induzida pela respiração lenta seria o principal mecanismo envolvido na redução da PA.

Estudo realizado no Laboratório de Hipertensão do Hospital das Clínicas da Faculdade de Medicina da Universidade de São Paulo (HC-FMUSP) comparou o uso do dispositivo de respiração guiada com o ato de escutar músicas calmas por 15 minutos, em uma única sessão, avaliando as respostas agudas da FR, atividade nervosa simpática periférica (ANSP) pela técnica da microneurografia, PA e frequência cardíaca (FC) em 27 voluntários com HA. Os resultados demonstram que houve diferença significativa em todas as variáveis analisadas para o grupo que utilizou o aparelho de respiração lenta guiada: FR, -11rpm; ANSP, -8 impulsos/min; PAS/PAD, -6/-4 mmHg e FC -2 bpm ( $p<0,05$ para todas as comparações). Já para o grupo controle, observou-se apenas reduções significativas $(p<0,05)$ para PAS/PAD, $-4 /-3 \mathrm{mmHg}$ e FC, -1 bpm; não havendo diferença para as outras variáveis: ANSP e FR ${ }^{25}$.

Em um relato de caso $^{26}$ também realizado pela equipe do Laboratório de Hipertensão HC-FMUSP foi demonstrada a eficácia do uso crônico do aparelho de respiração lenta guiada, durante 15 minutos diários por 8 semanas, em um paciente recém-diagnosticado com HA. Foi avaliada a PA de consultório, Monitorização Ambulatorial da Pressão arterial (MAPA) de 24 horas, catecolaminas plasmáticas e ANSP antes e após 8 semanas de tratamento. Houve uma discreta redução na PA de consultório (-6/-3 mmHg, PAS/PAD), PA de 24 horas (-4/-3 mmHg, PAS/PAD) e PA de vigília (-7/-3 mmHg, PAS/PAD) pela MAPA, mas não durante o sono $(+4 /+1 \mathrm{mmHg}$, PAS/PAD). Para as medidas da ANS, houve uma significante redução nos valores de noradrenalina, antes 
e após o período de tratamento (903 pg/ml versus $220 \mathrm{pg} / \mathrm{ml}$ ) bem como para a ANSP medida pela microneurografia (51 impulsos/minuto versus 22 impulsos/minuto).

Desta forma, o objetivo deste trabalho foi estudar a função autonômica de indivíduos hipertensos, particularmente a ANS, antes e após um período de intervenção de 8 semanas com o aparelho de respiração lenta guiada, reproduzindo a prática clínica. Espera-se dessa maneira, conseguir um melhor entendimento dos mecanismos envolvidos na redução da PA pela redução da FR, sendo a nossa hipótese, que se observe uma redução da atividade nervosa simpática com o uso regular e crônico do dispositivo de respiração lenta guiada. 
2. Objetivos 
Avaliar o efeito crônico da respiração lenta guiada em hipertensos sobre:

> a pressão arterial de consultório e MAPA de 24 horas;

$>$ a atividade nervosa simpática pela dosagem de catecolaminas plasmáticas e microneurografia. 
3. Métodos 


\section{1- Desenho do Estudo}

Ensaio clínico randomizado e aberto.

\section{2- Casuística}

Foram selecionados pacientes com hipertensão arterial em tratamento no Ambulatório de Hipertensão HC-FMUSP, além de hipertensos que ficaram cientes do estudo, por meio de cartazes, anúncios em jornais e revistas de pequena circulação, que preencheram os critérios de inclusão e não apresentaram nenhum dos critérios de exclusão.

\subsection{1- Critérios de inclusão}

$\checkmark$ Hipertensos, com ou sem uso de anti-hipertensivos, com média de PA de 24 horas pela MAPA acima dos limites de normalidade, PAS $>130 \mathrm{mmHg}$ e/ou PAD $>80 \mathrm{mmHg}^{27}$.

$\checkmark$ Adultos com idade $\geq 18$ anos.

$\checkmark$ Ambos os gêneros. 


\subsection{2- Critérios de exclusão}

$\checkmark$ Uso de medicações que interfiram na atividade nervosa simpática (Betabloqueadores e/ou Simpatolíticos de Ação Central).

$\checkmark$ Uso de 3 ou mais classes de anti-hipertensivos.

$\checkmark$ Presença de hipertensão arterial secundária excluída por meio de exames de triagem para a pesquisa de hipertensão renovascular, hiperaldosteronismo primário e feocromocitoma.

$\checkmark$ Insuficiência Renal Crônica (IRC) definido como Ritmo de Filtração Glomerular estimado pela fórmula do MDRD Study ${ }^{28}<60 \mathrm{ml} / \mathrm{min}$.

$\checkmark$ Doença Respiratória Crônica: antecedentes de asma brônquica ou doença pulmonar obstrutiva crônica.

$\checkmark$ Diabetes Mellitus definido por glicemia de jejum acima de $126 \mathrm{mg} / \mathrm{dl}$ e/ou hemoglobina glicada $>6,5 \%$ e/ou uso antidiabético orais/insulina.

$\checkmark$ Insuficiência Cardíaca Congestiva (ICC) definida por fração de ejeção $<55 \%$ (Método Teichholz) pelo Ecocardiograma.

$\checkmark$ Insuficiência Coronariana: antecedentes de angina pectoris/infarto agudo do miocárdio e/ou histórico de angioplastia de coronária/revascularização do miocárdio.

$\checkmark$ Índice de Massa Corporal (IMC) $>30 \mathrm{Kg} / \mathrm{m}^{2}$.

$\checkmark$ Gestantes 


\section{3- Métodos}

\subsection{1- Protocolo geral do estudo}

Os participantes foram selecionados no Ambulatório de Hipertensão do HC-FMUSP durante consulta médica de rotina e, posteriormente, foram encaminhados para uma entrevista no Laboratório de Hipertensão. Já os participantes hipertensos externos ao serviço passaram, inicialmente, por uma entrevista telefônica e, quando observado sua adequação ao protocolo, foram encaminhados para consulta médica.

Uma vez identificados os pacientes e aceitando estes participar do estudo, antes de iniciarem os experimentos foram esclarecidos a respeito de todos os procedimentos, riscos e benefícios envolvidos. Nessas condições assinaram o Termo de Consentimento Livre e Esclarecido (TCLE) - Anexo A. Este projeto, bem como as formas de divulgação utilizadas, foram aprovados pela Comissão de Ética para Análise de Projetos de Pesquisa do HC-FMUSP (№0107/10) - Anexo B.

Após assinatura do TCLE, todos os participantes foram submetidos à MAPA de 24 horas e a uma sessão experimental que visou avaliar a pressão arterial e a atividade nervosa simpática. Na sessão experimental foram verificados os mecanismos de regulação autonômica da PA em condições de repouso e em resposta ao estímulo físico isométrico com aparelho de preensão manual.

Posteriormente à realização da sessão experimental inicial, os participantes foram, aleatoriamente, divididos em dois grupos: controle ou 
respiração lenta. O esquema de aleatorização foi gerado por meio do site da internet randomization.com (http://www.randomization.com/), sendo 32 sujeitos divididos em 8 blocos, contendo 4 sujeitos aleatoriamente permutados por bloco, assegurando que um número igual de sujeitos fosse atribuído a cada tratamento cada vez que o número de indivíduos fosse um múltiplo do tamanho do bloco.

No período de intervenção, que teve duração de 8 semanas, os participantes do Grupo Respiração Lenta (GRL) fizeram uso do REPeRATE®, diariamente por 15 minutos com o objetivo de reduzir a frequência respiratória. Já os participantes do Grupo Controle (GC) utilizaram pelo mesmo período e tempo, um aparelho de MP3 que reproduzia músicas serenas. Durante essa fase do estudo, os participantes foram acompanhados após quatro semanas para checar a adesão às intervenções propostas.

Todos os participantes foram reavaliados após oito semanas de intervenção. Nessa reavaliação foram repetidas a MAPA e a sessão experimental que, neste momento, teve como objetivo verificar o efeito das intervenções (exercício de respiração lenta ou controle) sobre a pressão arterial e sua regulação autonômica.

Uma esquematização geral do estudo pode ser visualizada na Figura 1. A seguir cada procedimento será explicado de maneira mais detalhada. 


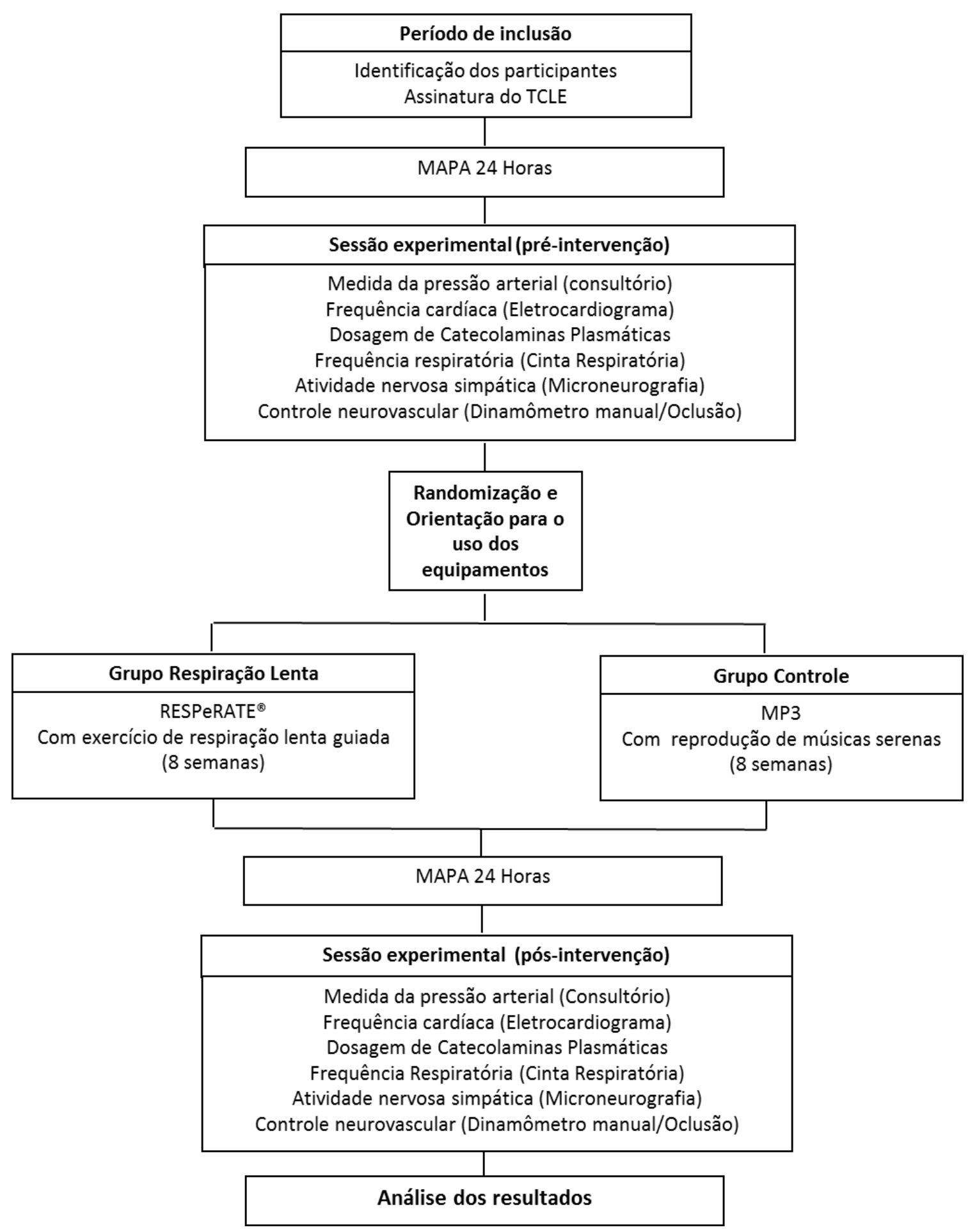

Figura 1 - Esquematização geral do estudo 


\subsection{2- Medidas da Pressão Arterial}

As medidas da pressão arterial foram realizadas de forma indireta (método oscilométrico) em duas ocasiões (pré e pós-intervenção) e com duas metodologias: MAPA de 24 horas e PA de consultório.

\subsubsection{Monitorização Ambulatorial da Pressão Arterial (MAPA)}

A MAPA foi realizada com aparelho Mobil-O-Graph NG (I.E.M., Solberg, Alemanha), validado de acordo com o protocolo da British Hypertension Society $^{29}$, programado para realizar medidas por 24 horas, sendo feitas a cada 20 minutos durante o período de vigília e sono, com tais períodos determinados de acordo com o diário de anotações do paciente. Os exames foram considerados válidos quando houve, pelo menos, 16 medidas no período de vigília e oito medidas no de sono. Além disso, foi respeitado um tempo mínimo de 22 horas de registro ${ }^{27}$. Caso estes critérios não fossem atingidos, o exame deveria ser repetido.

A MAPA foi instalada em dois momentos: após assinatura do TCLE e, novamente, após oito semanas de intervenção. Para isso, foi realizada a medida da circunferência do braço não dominante, e foi instalado um manguito de tamanho adequado à circunferência medida. O participante permaneceu com o aparelho durante aproximadamente 24 horas e então, foi retirado para análise. 


\subsubsection{Pressão arterial de consultório}

Para a medida da pressão arterial de consultório, foi utilizado o método automático oscilométrico de medida da pressão arterial por meio do monitor de pressão arterial DX 2710 (Dixtal Biomédica, Manaus, Brasil) previamente validado ${ }^{30}$

Estando o participante sentado, com o braço na altura do coração, foi posicionado no membro não dominante, um manguito de tamanho apropriado à circunferência do mesmo. Após cinco minutos em repouso foram realizadas três medidas da pressão arterial, respeitando-se um intervalo de dois minutos entre elas. Para determinação da pressão arterial do participante foi considerada a média das duas últimas medidas ${ }^{2}$.

\subsection{3- Frequência cardíaca}

Durante as sessões experimentais de análise da função autonômica, a FC foi monitorada pelo eletrocardiograma Dual Bio Amp (ADinstruments, Sidney, Austrália), durante todo o experimento e, em momentos específicos, a onda eletrocardiográfica foi digitalizada e registrada em microcomputador pelo programa LabChart 7 Pro (ADinstruments, Sidney, Austrália), numa frequência de amostragem de $2 \mathrm{~K} / \mathrm{s}$. 


\subsection{4- Dosagens de catecolaminas plasmáticas}

Para avaliação sistêmica da atividade nervosa simpática foi coletada uma amostra de sangue para dosagem de catecolaminas plasmáticas. Essa coleta foi obtida no Laboratório de Hipertensão do HC-FMUSP no mesmo dia em que foram realizadas as sessões experimentais pré e pós-intervenção.

Para coleta de catecolaminas plasmáticas, os participantes foram posicionados em decúbito dorsal e foi realizado punção de uma veia periférica por meio do Catéter Salf-T-Intima ${ }^{\mathrm{TM}}$ (BD, Utah, EUA). Após a punção da veia os participantes permaneceram em repouso por 30 minutos e após esse período a amostra de sangue foi coletada em um tubo com Ácido Etilenodiamino Tetra-Ácético (EDTA), que foi imediatamente acondicionado em gelo e, em seguida, encaminhado ao laboratório de análises clínicas para a dosagem de adrenalina, noradrenalina, dopamina pela técnica "High Performance Liquid Chromatography" (HPLC-ED).

\subsection{5- Respiração}

Os movimentos respiratórios foram monitorizados por uma cinta respiratória Piezoelétrica (Pneumotrace 2, UFI), que foi posicionada no tórax do participante. O sinal respiratório obtido por essa cinta foi amplificado e digitalizado pelo PowerLab 8/30 (ADinstruments, Sidney, Austrália) e registrado em microcomputador pelo programa LabChart 7 Pro (ADinstruments, Sidney, Austrália), numa frequência de amostragem de $1 \mathrm{~K} / \mathrm{s}$. 


\subsection{6- Atividade nervosa simpática periférica}

A atividade nervosa simpática periférica foi medida diretamente no fascículo do nervo fibular, por meio da técnica denominada microneurografia, que foi desenvolvida por Hagbarth e Vallbo ${ }^{31}$ e posteriormente descrita por Mark $^{32}$. Esta técnica possibilita o registro dos potenciais de ação das fibras A e C simpáticas em nervos periféricos.

A técnica consiste, inicialmente, em uma estimulação elétrica percutânea (40V a 120V) para mapear o trajeto do nervo. Assim, um microeletrodo de tungstênio (30-40 $\mathrm{mm}$ de comprimento, $0,2 \mathrm{~mm}$ de diâmetro e ponta não isolada de 1-5 $\mu \mathrm{m}$ ) foi impactado no nervo no local de melhor resposta à estimulação percutânea e, à aproximadamente $2 \mathrm{~cm}$ desse eletrodo, foi introduzido um outro para referência. Através do eletrodo ativo foram realizados ajustes finos da posição e uma estimulação interna de 4 - 5V até que se obteve um local onde esse estímulo deflagrasse contração involuntária no músculo da perna, sem parestesias. O sinal nervoso desse local foi amplificado, filtrado e integrado pelo PowerLab 8/30 (ADinstruments, Sidney, Austrália) e registrado durante 20 minutos em microcomputador pelo programa LabChart 7 Pro (ADinstruments, Sidney, Austrália), numa frequência de amostragem de $2 \mathrm{~K} / \mathrm{s}$ (Figura 2).

A atividade nervosa simpática foi medida, contando-se o número de impulsos nervosos por minuto. 

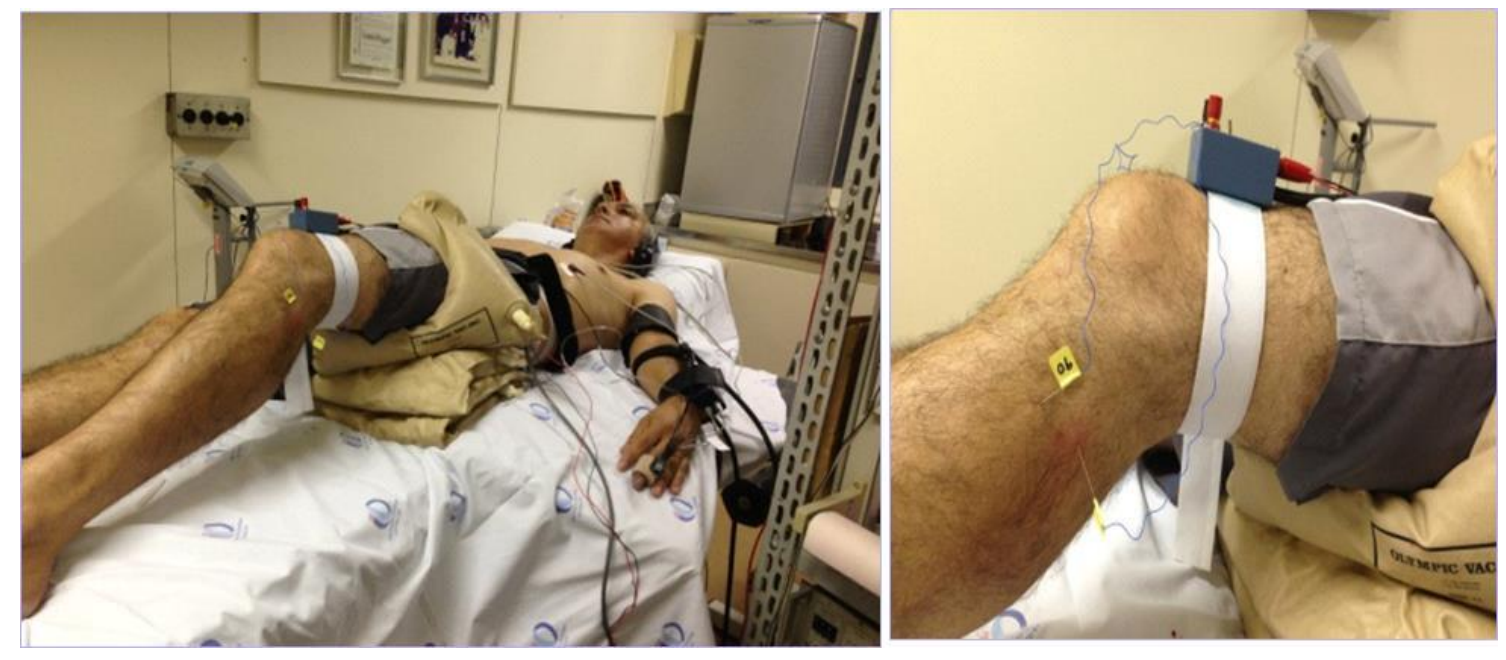

Figura 2 - Técnica de microneurografia durante sessão experimental: posicionamento do eletrodo ativo e referência na perna esquerda de um participante

\subsection{7- Controle neurovascular}

O controle neurovascular foi testado durante as sessões experimentais pré e pós-intervenção, com a realização de manobras de estimulação da atividade nervosa simpática. Para este procedimento foi utilizado o dinamômetro manual (Baseline®, Nova lorque, EUA).

No início da sessão experimental, foram realizadas as medidas da contração voluntária máxima do antebraço do membro dominante com o auxílio do dinamômetro manual. Foram permitidas três tentativas, respeitando-se um intervalo mínimo de um minuto entre elas.

A partir do décimo minuto de registro da ANSP da sessão experimental pré e pós-intervenção foi realizado o estímulo físico isométrico com o dinamômetro manual, por 3 minutos numa intensidade equivalente a $30 \%$ da 
contração voluntária máxima, possibilitando analisar o mecanorreflexo ${ }^{33}$. Em seguida, imediatamente após a finalização do estímulo físico isométrico, foi efetuada a oclusão da circulação do braço por 2 minutos, com o auxílio de um manguito, a uma pressão de $200 \mathrm{mmHg}$, possibilitando, neste momento, analisar a participação do metaborreflexo ${ }^{33}$ (Figura 3).

A análise de ambos os controles neurovasculares foi realizada a partir do comportamento da atividade nervosa simpática periférica frente às manobras excitatórias.

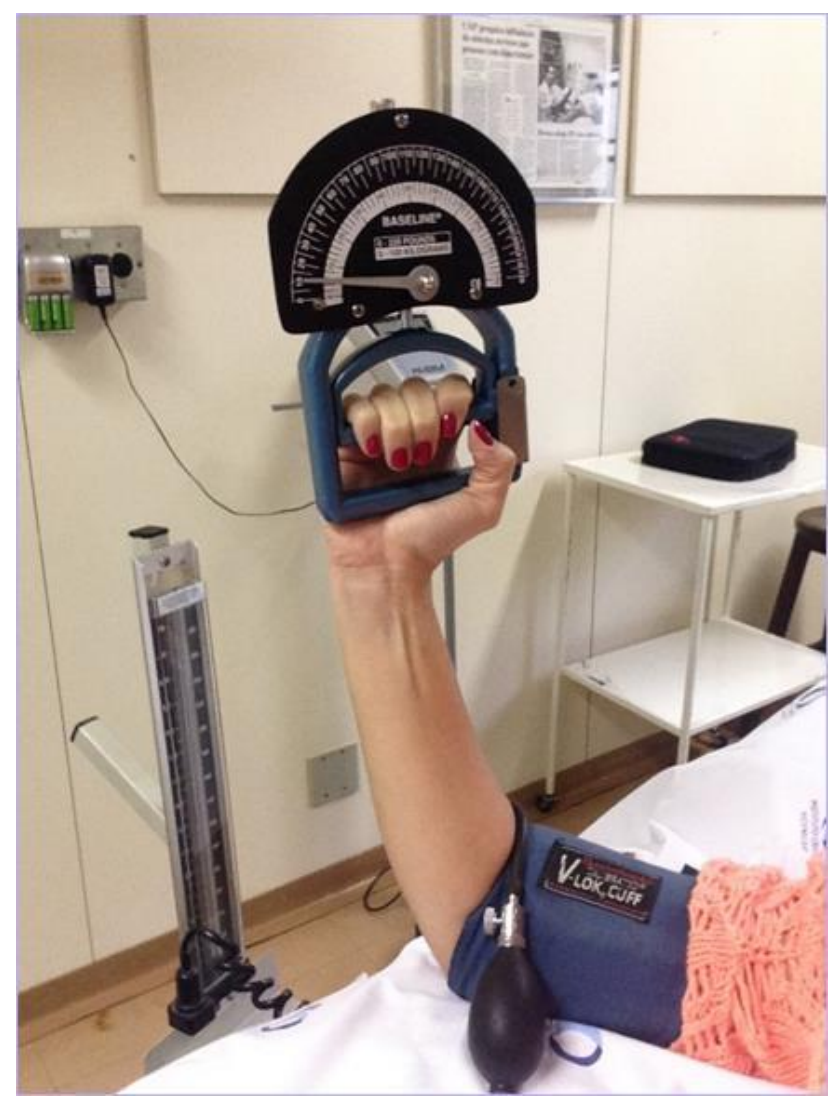

Figura 3 - Estímulo físico isométrico durante sessão experimental: participante realizando a preensão manual com o dinamômetro 


\subsection{8- Intervenções}

A intervenção proposta neste estudo para o Grupo Respiração Lenta (GRL) foi realizada por meio da utilização do equipamento comercializado como RESPeRATE ${ }^{\circledR}$ (Intercure, Lod, Israel). Esse equipamento é composto por fone de ouvido, cinta elástica com sensor respiratório e uma unidade computadorizada que, com o auxílio de uma melodia, é capaz de guiar o voluntário a uma frequência respiratória equivalente a dez incursões respiratórias por minuto ou menos (Figura 4). De forma sucinta, o equipamento, inicialmente, monitora a respiração do indivíduo e, em seguida personaliza uma melodia composta por dois tons diferentes, um para inspiração e outro para expiração. O participante foi instruído a seguir a orientação visual de inspiração e expiração localizada na tela do equipamento, que ficou disponível durante todo o exercício respiratório, sincronizando assim sua respiração com a melodia proposta pelo equipamento que, gradualmente, prolongou o tom de expiração, induzindo o participante a respirar mais lentamente.

Para o Grupo Controle (GC) foi disponibilizado um aparelho de MP3 que reproduziu músicas serenas previamente gravadas pelo pesquisador.

Os participantes foram orientados verbalmente e por escrito quanto ao manuseio do equipamento, de acordo com cada grupo, devendo utilizá-lo por 15 minutos diários durante 8 semanas. 


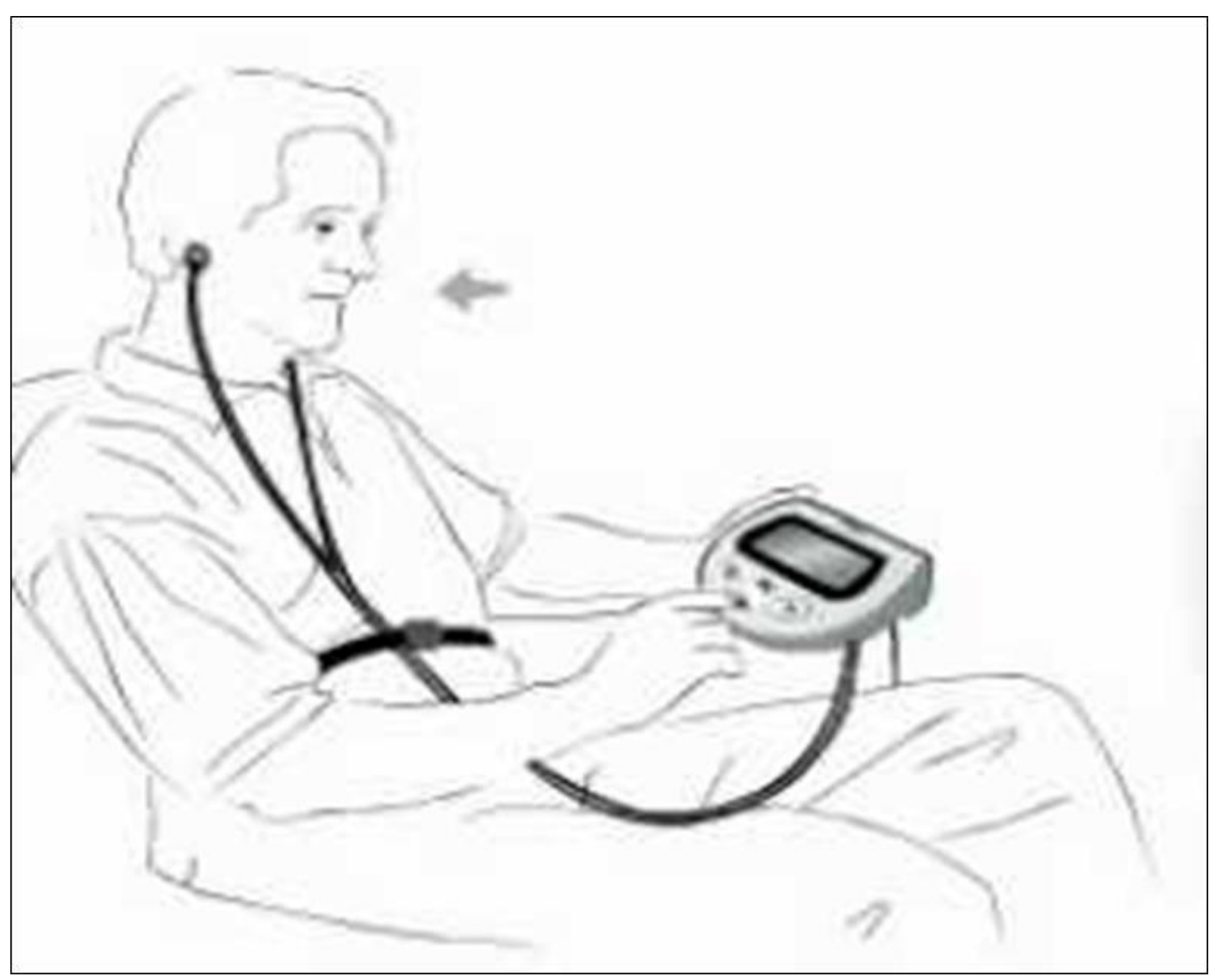

Figura 4 - RESPeRATE ${ }^{\circledR}$ (Intercure, Lod, Israel)

\subsection{9- Adesão à Intervenção}

Para a avaliação da adesão a intervenção com o aparelho de respiração lenta guiada e com o aparelho de MP3, foi entregue ao participante um impresso com um calendário referente ao período de oito semanas em que deveriam realizar os procedimentos propostos durante a randomização, ou seja, exercício de respiração lenta ou somente ouvir a melodia produzida pelo MP3. Nesse impresso os participantes fizeram um diário assinalando o dia e hora em que foi realizado o procedimento e sua duração. 
Esse diário foi apresentado ao pesquisador a cada 30 dias e entregue juntamente com o aparelho, no momento em que foi realizada a sessão experimental pós-intervenção.

Além disso, para o GRL, foi feita a descarga, a cada 30 dias, dos dados armazenados na memória do RESPeRATE ${ }^{\circledR}$, que continham o número de sessões realizadas; a média do tempo gasto (minutos) na zona de respiração terapêutica (<10 respirações/minuto); a média da frequência respiratória inicial; a média da frequência respiratória final; a média do tempo em que a frequência respiratória esteve sincronizada com os tons guias (\%) e a média de tempo em que o sensor respiratório estava apto a identificar a respiração (\%).

\section{4- Tamanho da Amostra}

Considerando-se:

a) um nível de significância de 5\%;

b) um poder do teste de $80 \%$;

c) teste de hipótese: monocaudal;

d) conhecendo-se o desvio-padrão das medidas de microneurografia de cerca 7 impulsos/minuto;

e) esperando-se uma diferença entre os grupos (respiração guiada e controle) de 8 impulsos/minuto de redução da atividade nervosa simpática pela microneurografia (dados de literatura ${ }^{25}$ ), chegou-se a um número de 10 participantes em cada grupo de estudo, perfazendo um total de 20 participantes. 


\section{5- Análise estatística}

Para atender aos pressupostos da análise estatística de dados paramétricos e não paramétricos foi utilizado o pacote estatístico STATA (versão 13.0).

Os dados foram avaliados quanto à normalidade por meio do teste de Shapiro-Wilk e quanto à homogeneidade de variância pelo teste de FlignerKilleen, de acordo com níveis das variáveis de tratamento e fase.

As variáveis categóricas foram descritas como número absoluto e percentual relativo. Já as variáveis de distribuição normal foram mostradas como média \pm desvio padrão e as de distribuição não normal como mediana e $1^{\circ}$ e $3^{\circ}$ quartis.

Para análise das variáveis categóricas do perfil da amostra, utilizou-se o teste exato de Fisher. Os dados dos dois grupos foram comparados via análise de variância em um fator com medidas repetidas, tendo o tratamento como efeito entre os sujeitos e a fase como efeito intra-sujeitos. Para a localização das diferenças, foi utilizado o teste de Welch pareados para cada combinação de tratamento e fase, com correção de Holm para comparações múltiplas quando necessário.

As diferenças foram consideradas significativas quando $p<0,05$. 


\section{Resultados}




\section{1- Características da amostra}

O recrutamento dos participantes ocorreu conforme mostrado no Fluxograma da Figura 5. Dezessete participantes no GRL e 15 participantes no GC chegaram ao fim do protocolo de estudo, sendo que 10 participantes de cada grupo tiveram o registro da atividade nervosa simpática periférica, realizada pela técnica da microneurografia, antes e após o período de intervenção bem sucedido.

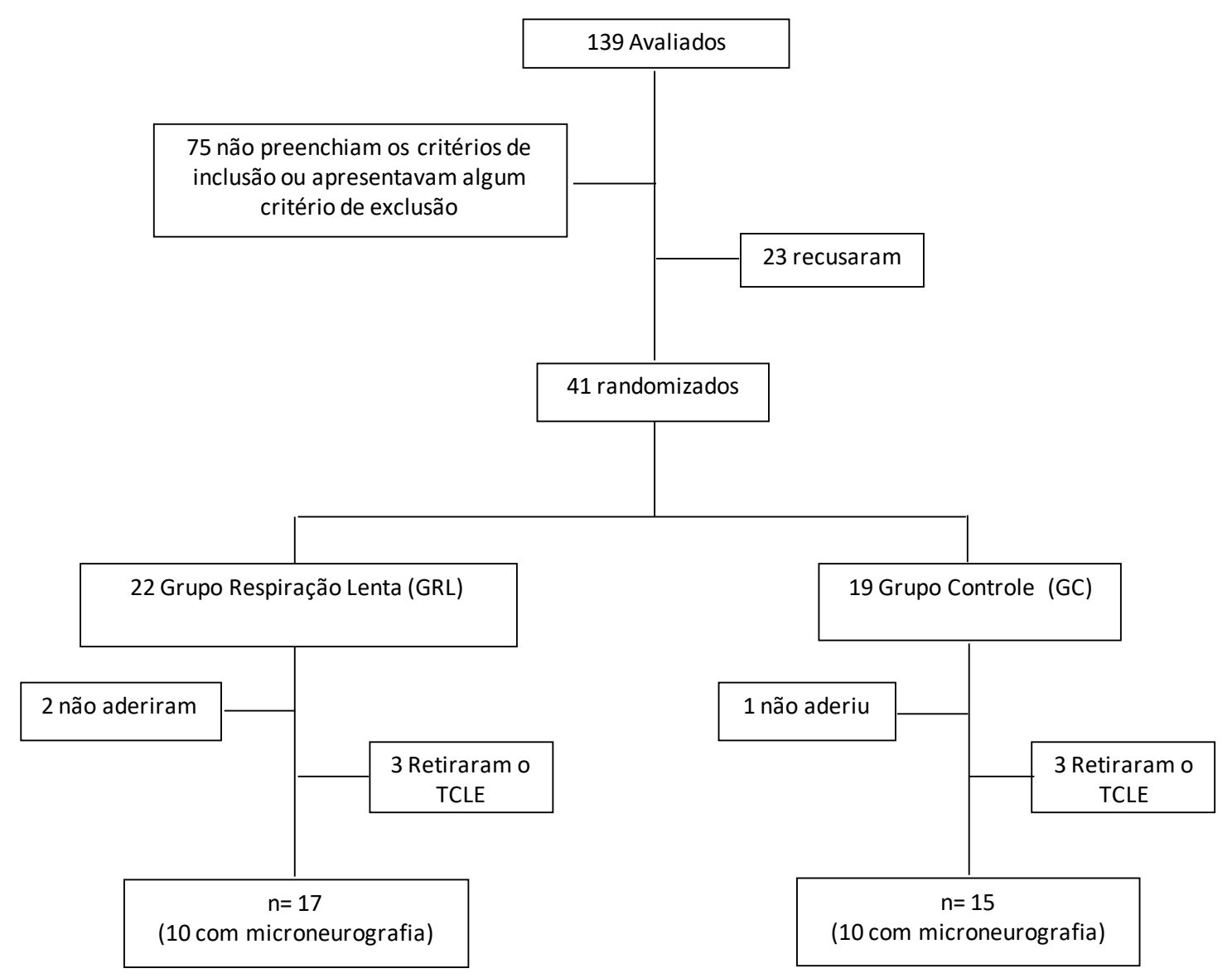

Figura 5 - Fluxograma de entrada e saída dos participantes durante o estudo 
As características clínicas e demográficas dos participantes que terminaram o protocolo estão expostas na Tabela 1. Conforme se pode notar, não houve diferença entre os grupos na maioria das variáveis, com exceção do tratamento medicamentoso, onde um percentual maior dos participantes do GC fazia uso de medicamentos anti-hipertensivos.

Tabela 1 - Características demográficas, clínicas e laboratoriais dos grupos respiração Lenta (GRL) e controle (GC)

\begin{tabular}{lccc}
\hline \multicolumn{1}{c}{ Variável (Média \pm DP ou \%) } & GRL (n=17) & GC (n=15) & $\mathbf{p}$ \\
\hline Idade (anos) & $49 \pm 9$ & $52 \pm 11$ & 0,40 \\
Gênero \% (F) & 47 & 73 & 0,16 \\
Circunferência Abdominal & $90 \pm 9$ & $91 \pm 9$ & 0,78 \\
IMC (Kg/m ${ }^{2}$ ) & $25,8 \pm 3,0$ & $27,1 \pm 2,4$ & 0,18 \\
Hemoglobina (g/dL) & $14,3 \pm 1,3$ & $13,9 \pm 1,4$ & 0,19 \\
Colesterol Total (mg/dL) & $207 \pm 51$ & $196 \pm 30$ & 0,14 \\
HDL (mg/dL) & $60 \pm 14$ & $57 \pm 12$ & 0,48 \\
LDL (mg/dL) & $124 \pm 45$ & $116 \pm 25$ & 0,18 \\
Triglicerídeos (mg/dL) & $124 \pm 91$ & $114 \pm 49$ & 0,50 \\
Creatinina (mg/dL) & $0,87 \pm 0,17$ & $0,89 \pm 0,18$ & 0,92 \\
RFG (mI/min) & $93 \pm 13$ & $88 \pm 18$ & 0,42 \\
Potássio (mEq/L) & $4,3 \pm 0,4$ & $4,2 \pm 0,5$ & 0,89 \\
Glicemia jejum (mg/dL) & $90 \pm 14$ & $86 \pm 10$ & 0,20 \\
Hemoglobina Glicada (\%) & $5,5 \pm 0,3$ & $5,4 \pm 0,3$ & 0,28 \\
PAS de consultório (mmHg) & $134 \pm 12$ & $131 \pm 9$ & 0,68 \\
PAD de consultório (mmHg) & $87 \pm 9$ & $84 \pm 9$ & 0,31 \\
Uso de Anti-hipertensivos (Sim/Não) & $4 / 13$ & $12 / 3$ & $\mathrm{p}<0,01$ \\
\hline
\end{tabular}

IMC= Índice de Massa Corpóreo; HDL= Lipoproteína de Alta Densidade; LDL= Lipoproteína de Baixa Densidade; $T G=$ Triglicérides; $R F G=$ Ritimo de filtração Glomerular; $P A S=$ Pressão Arterial Sistólica; PAD= Pressão Arterial Diastólica 


\section{2- Pressão arterial de consultório, MAPA de 24 horas e frequência cardíaca}

A Tabela 2 mostra os valores de pressão arterial de consultório, MAPA de 24 horas e frequência cardíaca, nos períodos pré e pós-intervenção, dos grupos controle e respiração lenta.

Tabela 2 - Comportamento da pressão arterial de consultório e MAPA de 24 horas antes e após a intervenção, de acordo com a randomização. Grupo Respiração Lenta (GRL) versus Grupo Controle (GC)

\begin{tabular}{lcccc}
\hline \multirow{2}{*}{\multicolumn{1}{c}{ Variável }} & \multicolumn{2}{c}{ GRL (n=17) } & \multicolumn{2}{c}{ GC (n=15) } \\
\cline { 2 - 5 } & Pré & Pós & Pré & Pós \\
\hline PAS de consultório (mmHg) & $134 \pm 12$ & $134 \pm 15$ & $131 \pm 9$ & $133 \pm 11$ \\
PAD de consultório (mmHg) & $87 \pm 9$ & $88 \pm 12$ & $84 \pm 9$ & $84 \pm 6$ \\
PAS MAPA 24 hs (mmHg) & $127 \pm 8$ & $130 \pm 11$ & $128 \pm 8$ & $126 \pm 11$ \\
PAD MAPA 24 hs (mmHg) & $88 \pm 5$ & $90 \pm 7$ & $89 \pm 7$ & $86 \pm 8$ \\
PAS MAPA Vigília (mmHg) & $131 \pm 8$ & $134 \pm 11$ & $131 \pm 10$ & $128 \pm 10 \#$ \\
PAD MAPA Vigília (mmHg) & $92 \pm 5$ & $94 \pm 7$ & $92 \pm 9$ & $88 \pm 8 \#$ \\
PAS MAPA Sono (mmHg) & $118 \pm 10$ & $120 \pm 12$ & $122 \pm 9$ & $122 \pm 15$ \\
PAD MAPA Sono (mmHg) & $78 \pm 7$ & $80 \pm 9$ & $82 \pm 8$ & $80 \pm 10$ \\
Frequência Cardíaca de 24 horas (bpm) & $75 \pm 7$ & $78 \pm 7^{*}$ & $77 \pm 8$ & $76 \pm 9$ \\
Frequência Cardíaca de vigília (bpm) & $80 \pm 7$ & $83 \pm 8$ & $81 \pm 9$ & $81 \pm 9$ \\
Frequência Cardíaca de sono (bpm) & $66 \pm 8$ & $70 \pm 9^{*}$ & $68 \pm 8$ & $68 \pm 9$ \\
\hline
\end{tabular}

PAS= Pressão Arterial Sistólica; $P A D=$ Pressão Arterial Diastólica; MAPA= Monitorização Ambulatorial da Pressão Arterial; bpm= batimentos por minuto.

* $p<0,05$ vs GRL Pré

$\# p<0,05$ vs GC Pré e GRL Pós 
Não houve mudança na PA de consultório antes e após a intervenção nos dois grupos.

Observou-se redução na pressão arterial sistólica (PAS) e diastólica (PAD) na vigília entre os períodos pré e pós-intervenção apenas no GC $(131 \pm 10 / 92 \pm 9$ vs $128 \pm 10 / 88 \pm 8 \mathrm{mmHg}, \mathrm{p}<0,05)$

Para os parâmetros de frequência cardíaca avaliados pela MAPA, houve uma elevação significativa desta variável entre os períodos pré e pós intervenção do GRL nas 24 horas $(75 \pm 7$ vs $78 \pm 7$ bpm, $p<0,05)$ e no sono $(66 \pm 8$ vs $70 \pm 9$ bpm, $p<0,05)$.

\section{3- Atividade nervosa simpática - concentração de noradrenalina plasmática}

As concentrações de noradrenalina plasmática, nos períodos pré e pósintervenção, de acordo com o grupo de estudo, controle e respiração lenta, podem ser visualizadas graficamente na Figura 6.

Não foi observada diferença estatisticamente significante na concentração de noradrenalina plasmática entre o GC e GRL na fase préintervenção (221(170-266) vs 227 (193-309) pg/ml, p= 0,97). Também não foram observadas diferenças entre as concentrações plasmáticas de noradrenalina entre os períodos pré e pós-intervenção, tanto no GC (221(170-266) vs 242 (179-337) pg/ml, p=0,54), como no GRL (227 (193-309) vs 211 (153-269) $\mathrm{pg} / \mathrm{ml}, \mathrm{p}=0,54)$. 


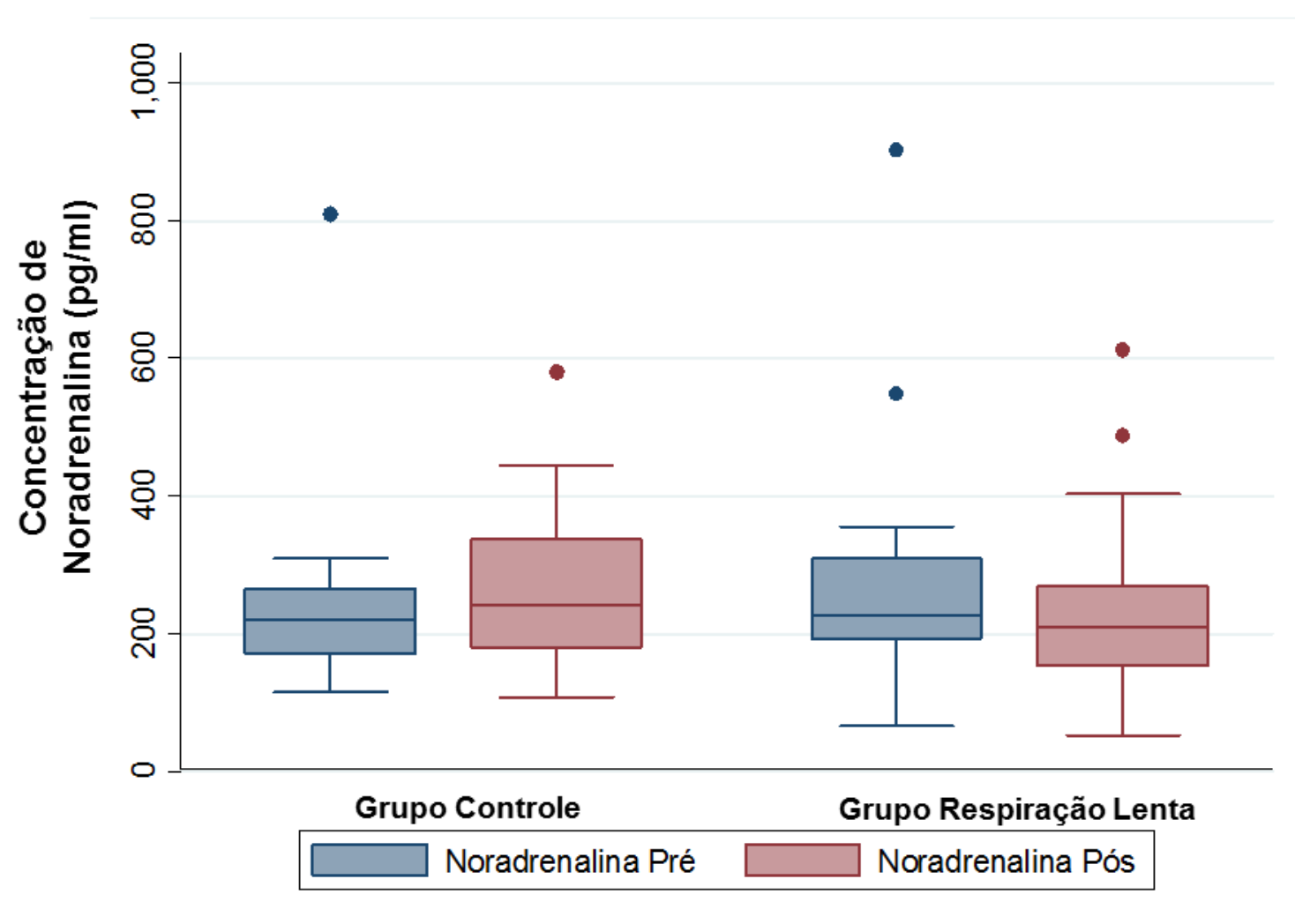

$p>0,05$; noradrenalina pré vs noradrenalina pós, para o grupo controle e grupo respiração lenta

Figura 6 - Concentração de noradrenalina pré e pós intervenção, nos grupos controle e respiração lenta

A Tabela 3 mostra a análise dos dados dividindo os participantes de acordo com a atividade nervosa simpática basal determinada pela mediana da concentração de noradrenalina plasmática. Da mesma forma que observado anteriormente, não se observou alterações significativas da pressão arterial, seja de consultório ou todas as médias de PA pela MAPA, bem como da concentração de noradrenalina plasmáticas entre os grupos após a intervenção, independentemente da atividade nervosa simpática basal. 
Tabela 3 - Pressão arterial (Consultório e MAPA de 24 horas) e concentração de noradrenalina plasmática, antes e após a intervenção, de acordo com a randomização e atividade nervosa simpática basal (mediana da noradrenalina plasmática). Grupo Respiração Lenta (GRL) versus Grupo Controle (GC)

\begin{tabular}{|c|c|c|c|c|c|c|c|c|c|c|c|c|}
\hline \multirow{3}{*}{ Variável } & \multicolumn{6}{|c|}{$-50 \%$} & \multicolumn{6}{|c|}{$+50 \%$} \\
\hline & \multicolumn{3}{|c|}{ GRL $(n=8)$} & \multicolumn{3}{|c|}{$\mathrm{GC}(\mathrm{n}=8)$} & \multicolumn{3}{|c|}{ GRL $(n=9)$} & \multicolumn{3}{|c|}{ GC $(n=7)$} \\
\hline & Pré & Pós & $p$ & Pré & Pós & $p$ & Pré & Pós & $p$ & Pré & Pós & $p$ \\
\hline $\begin{array}{l}\text { PAS consultório } \\
(\mathrm{mmHg})\end{array}$ & $131 \pm 13$ & $129 \pm 16$ & 0,51 & $130 \pm 10$ & $132 \pm 13$ & 0,59 & $136 \pm 12$ & $138 \pm 14$ & 0,79 & $134 \pm 9$ & $134 \pm 8$ & 1,00 \\
\hline $\begin{array}{l}\text { PAD consultório } \\
(\mathrm{mmHg})\end{array}$ & $86 \pm 10$ & $84 \pm 15$ & 0,41 & $84 \pm 7$ & $86 \pm 7$ & 0,46 & $88 \pm 8$ & $91 \pm 8$ & 0,13 & $85 \pm 11$ & $83 \pm 6$ & 0,52 \\
\hline $\begin{array}{l}\text { PAS MAPA } 24 \text { hs } \\
(\mathrm{mmHg})\end{array}$ & $128 \pm 9$ & $129 \pm 9$ & 0,69 & $128 \pm 9$ & $124 \pm 8$ & 0,08 & $127 \pm 7$ & $131 \pm 13$ & 0,26 & $129 \pm 7$ & $128 \pm 14$ & 0,80 \\
\hline $\begin{array}{l}\text { PAD MAPA } 24 \text { hs } \\
(\mathrm{mmHg})\end{array}$ & $88 \pm 6$ & $89 \pm 7$ & 0,38 & $90 \pm 6$ & $87 \pm 5$ & 0,15 & $88 \pm 3$ & $90 \pm 7$ & 0,30 & $88 \pm 9$ & $84 \pm 10$ & 0,19 \\
\hline $\begin{array}{l}\text { PAS MAPA Vigília } \\
(\mathrm{mmHg})\end{array}$ & $132 \pm 9$ & $134 \pm 9$ & 0,49 & $131 \pm 11$ & $126 \pm 8$ & 0,05 & $130 \pm 8$ & $135 \pm 13$ & 0,23 & $132 \pm 9$ & $131 \pm 11$ & 0,71 \\
\hline $\begin{array}{l}\text { PAD MAPA Vigília } \\
(\mathrm{mmHg})\end{array}$ & $92 \pm 6$ & $94 \pm 7$ & 0,13 & $93 \pm 7$ & $89 \pm 7$ & 0,07 & $92 \pm 6$ & $95 \pm 7$ & 0,36 & $91 \pm 12$ & $87 \pm 9$ & 0,26 \\
\hline $\begin{array}{l}\text { PAS MAPA Sono } \\
(\mathrm{mmHg})\end{array}$ & $119 \pm 12$ & $118 \pm 11$ & 0,78 & $121 \pm 9$ & $121 \pm 9$ & 0,91 & $118 \pm 9$ & $122 \pm 13$ & 0,35 & $122 \pm 10$ & $124 \pm 20$ & 0,73 \\
\hline $\begin{array}{l}\text { PAD MAPA Sono } \\
(\mathrm{mmHg})\end{array}$ & $79 \pm 11$ & $79 \pm 10$ & 1,00 & $84 \pm 7$ & $82 \pm 5$ & 0,61 & $78 \pm 4$ & $81 \pm 9$ & 0,28 & $81 \pm 9$ & $78 \pm 14$ & 0,35 \\
\hline $\begin{array}{l}\text { Noradrenalina } \\
(\mathrm{pg} / \mathrm{ml})\end{array}$ & $168 \pm 62$ & $166 \pm 137$ & 0,97 & $176 \pm 35$ & $210 \pm 71$ & 0,31 & $391 \pm 212$ & $299 \pm 135$ & 0,36 & $340 \pm 209$ & $331 \pm 142$ & 0,85 \\
\hline
\end{tabular}

$\mathrm{PAS}=$ Pressão Arterial Sistólica; $\mathrm{PAD}=$ Pressão Arterial Diastólica; MAPA= Monitorização Ambulatorial da Pressão Arterial. 
A análise dos dados apresentados a seguir se referem apenas aos participantes que chegaram ao final do estudo com o registro da ANSP pré e pós-intervenção, avaliado por meio da técnica da microneurografia bem sucedida ( $n=20)$, sendo 10 no GC e 10 no GRG.

\section{4- Características da amostra - subgrupo dos participantes com microneurografia}

As características clínicas e demográficas dos participantes com microneurografia estão expostas na Tabela 4. Observa-se homogeneidade entre os grupos para a grande maioria das variáveis, exceto para o gênero, que foi composto por $80 \%$ de mulheres no $G C(p=0,02)$. 
Tabela 4 - Características demográficas, clínicas e laboratoriais dos grupos controle (GC) e respiração lenta (GRL); subgrupo dos voluntários com microneurografia

\begin{tabular}{lccc}
\hline \multicolumn{1}{c}{ Variável (Média \pm DP ou \%) } & GRL $(\mathbf{n}=\mathbf{1 0})$ & $\mathbf{G C}(\mathbf{n}=\mathbf{1 0})$ & $\mathbf{p}$ \\
\hline Idade (anos) & $49 \pm 6$ & $52 \pm 12$ & 0,50 \\
Gênero \% (F) & 20 & 80 & 0,02 \\
Circunferência Abdominal & $92 \pm 9$ & $92 \pm 8$ & 0,97 \\
IMC (Kg/m ${ }^{2}$ ) & $26,7 \pm 2,5$ & $27,1 \pm 2,6$ & 0,75 \\
Hemoglobina (g/dL) & $14,4 \pm 1,4$ & $13,9 \pm 1,4$ & 0,34 \\
Colesterol total (mg/dL) & $206 \pm 62$ & $191 \pm 32$ & 0,19 \\
HDL (mg/dL) & $53 \pm 13$ & $59 \pm 12$ & 0,52 \\
LDL (mg/dL) & $127 \pm 53$ & $109 \pm 26$ & 0,12 \\
Triglicerídeos (mg/dL) & $147 \pm 113$ & $117 \pm 57$ & 0,36 \\
Creatinina (mg/dL) & $0,93 \pm 0,20$ & $0,89 \pm 0,17$ & 0,29 \\
RFG (ml/min) & $94 \pm 16$ & $88 \pm 22$ & 0,73 \\
Potássio (mEq/L) & $4,4 \pm 0,4$ & $4,3 \pm 0,5$ & 0,60 \\
Glicemia Jejum (mg/dL) & $87 \pm 13$ & $85 \pm 8$ & 0,60 \\
Hemoglobina Glicada (\%) & $5,5 \pm 0,4$ & $5,4 \pm 0,3$ & 0,26 \\
PAS de consultório (mmHg) & $137 \pm 12$ & $134 \pm 8$ & 0,57 \\
PAD de consultório (mmHg) & $89 \pm 9$ & $87 \pm 6$ & 0,39 \\
Uso de anti-hipertensivos (Sim/Não) & $3 / 7$ & $8 / 2$ & 0,07 \\
\hline
\end{tabular}

IMC= Índice de Massa Corpóreo; HDL= Lipoproteína de Alta Densidade; LDL= Lipoproteína de Baixa Densidade; RFG= Ritimo de filtração Glomerular; PAS= Pressão Arterial Sistólica; PAD= Pressão Arterial Diastólica.

\section{5- Pressão arterial de consultório, MAPA de 24 horas e frequência cardíaca - subgrupo dos participantes com microneurografia}

Os dados da Tabela 5 mostram os valores da pressão arterial de consultório, MAPA de 24 horas e frequência cardíaca, nos períodos pré e pósintervenção, dos Grupos Controle e Respiração Lenta do subgrupo dos participantes com microneurografia. 
Tabela 5 - Comportamento da pressão arterial de consultório e pela MAPA de 24 horas antes e após a intervenção de acordo com a randomização, Grupo Respiração Lenta (GRL) versus Grupo Controle (GC); subgrupo dos voluntários com microneurografia

\begin{tabular}{lcccc}
\hline \multirow{2}{*}{ Variável } & \multicolumn{2}{c}{ GRL $(\mathbf{n = 1 0})$} & \multicolumn{2}{c}{ GC $(\mathbf{n = 1 0})$} \\
\cline { 2 - 5 } & Pré & Pós & Pré & Pós \\
\hline PAS de consultório (mmHg) & $137 \pm 12$ & $138 \pm 17$ & $134 \pm 8$ & $134 \pm 12$ \\
PAD de consultório (mmHg) & $89 \pm 9$ & $90 \pm 13$ & $87 \pm 6$ & $86 \pm 5$ \\
PAS MAPA 24 hs (mmHg) & $127 \pm 9$ & $129 \pm 8$ & $127 \pm 8$ & $124 \pm 10$ \\
PAD MAPA 24 hs (mmHg) & $88 \pm 6$ & $90 \pm 7$ & $88 \pm 7$ & $85 \pm 5$ \\
PAS MAPA Vigília (mmHg) & $132 \pm 10$ & $133 \pm 9$ & $130 \pm 10$ & $127 \pm 10$ \\
PAD MAPA Vigília (mmHg) & $93 \pm 7$ & $95 \pm 8$ & $91 \pm 9$ & $88 \pm 6$ \\
PAS MAPA Sono (mmHg) & $117 \pm 12$ & $120 \pm 9$ & $121 \pm 10$ & $118 \pm 11$ \\
PAD MAPA Sono (mmHg) & $78 \pm 9$ & $80 \pm 10$ & $83 \pm 6$ & $79 \pm 4 *$ \\
Frequência Cardíaca de 24 horas & $77 \pm 7$ & $80 \pm 8$ & $77 \pm 7$ & $77 \pm 8$ \\
Frequência Cardíaca de vigília & $81 \pm 8$ & $85 \pm 8 \#$ & $80 \pm 7$ & $81 \pm 9$ \\
Frequência Cardíaca de sono & $67 \pm 10$ & $70 \pm 10$ & $69 \pm 8$ & $69 \pm 9$ \\
\hline
\end{tabular}

PAS $=$ Pressão Arterial Sistólica; PAD= Pressão Arterial Diastólica.

* $\mathrm{p}<0,05$ vs GC Pré

$\# p<0,05$ vs GRG Pré

Em relação ao comportamento da pressão arterial de consultório, não foram observadas diferenças estatisticamente significativas entre os grupos ou entre as fases pré e pós-intervenção.

$\mathrm{Na}$ avaliação da PA medida pela MAPA, observa-se que o GC apresentou redução significativa da PAD de sono entre os períodos pré e pós intervenção ( $83 \pm 6$ vs $79 \pm 4 \mathrm{mmHg}, \mathrm{p}<0,05)$.

Como pode ser observado, houve um aumento na frequência cardíaca de vigília no GRL entre as fases pré e pós-intervenção ( $81 \pm 8$ vs $85 \pm 8$ bpm, $p<0,05)$, mas o mesmo não ocorreu no GC. 


\section{6- Atividade nervosa simpática - Concentração de noradrenalina plasmática e microneurografia}

A Figura 7 exibe o gráfico das concentrações de noradrenalina plasmáticas, nos períodos pré e pós-intervenção, de acordo com o grupo de estudo, controle e respiração lenta, no subgrupo dos participantes com microneurografia.

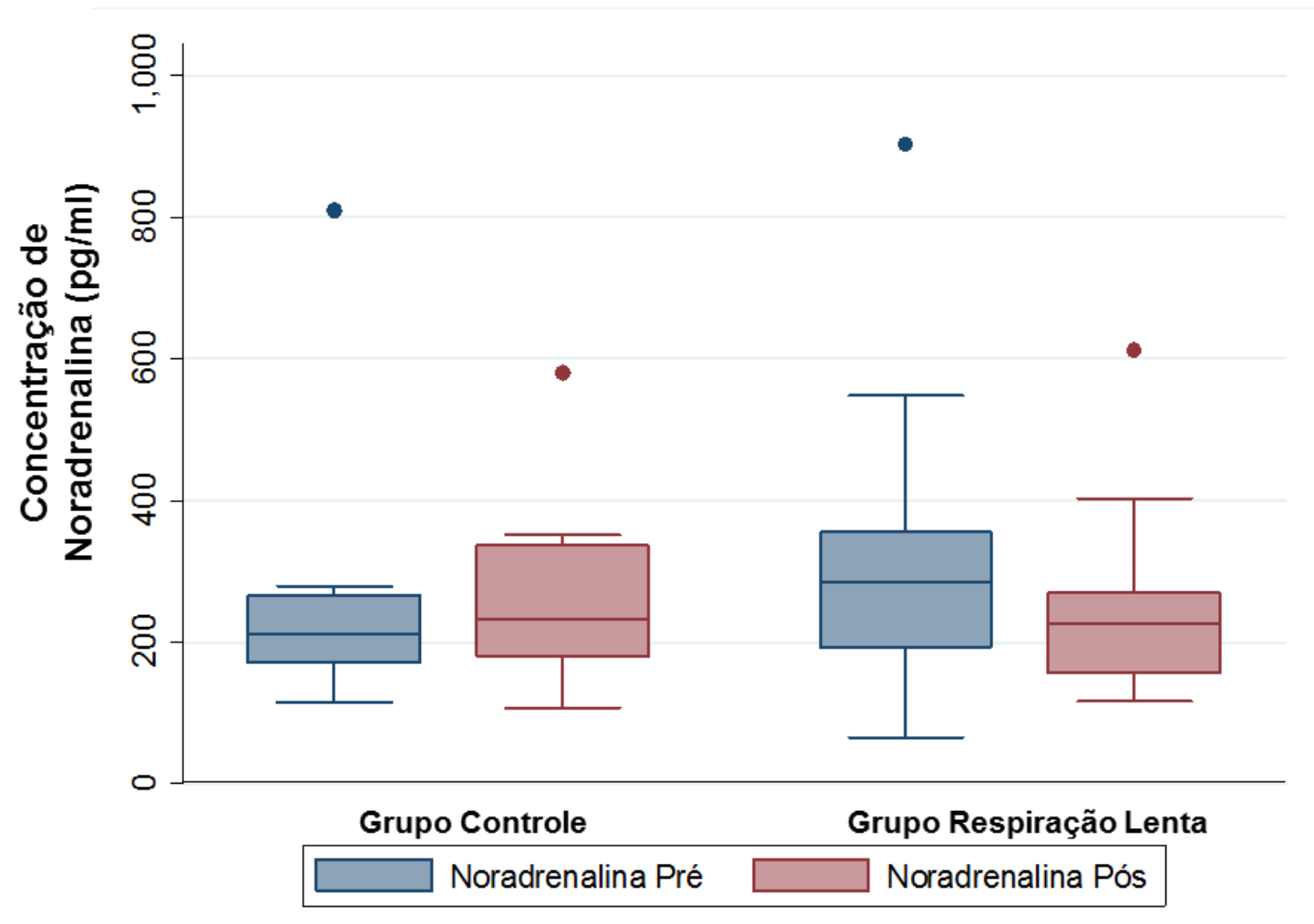

p>0,05; noradrenalina pré vs noradrenalina pós, para o grupo controle e grupo respiração lenta.

Figura 7 - Concentração de noradrenalina pré e pós intervenção, nos grupos controle e respiração lenta; subgrupo dos participantes com microneurografia

Não foram observadas diferenças significativas, do ponto de vista estatístico, nas concentrações de noradrenalina plasmáticas entre o GC e GRL na 
fase pré-intervenção (211(170-266) vs $285(193-356) \mathrm{pg} / \mathrm{ml}, \mathrm{p}=0,71)$ ou entre as fases pré e pós-intervenção, tanto no GC (221(170-266) vs 231 (179-337) pg/ml, $\mathrm{p}=0,51)$, como no GRL (285 (193-356) vs 227 (156-269) pg/ml, p= 0,51).

Na avaliação da ANSP basal medida pela técnica da microneurografia, observou-se aumento, estatisticamente significativo, no número de impulsos/minuto entre os períodos pré e pós-intervenção, tanto para o GRL (16 \pm 6 vs $22 \pm 8$ impulsos/min, $p<0,05)$ como para o GC $(20 \pm 5$ vs $23 \pm 5, p<0,05)$. O comportamento da atividade nervosa simpática periférica de cada participante individualmente, pré e pós-intervenção, dos grupos controle e respiração lenta, pode ser visualizado graficamente na Figura 8.

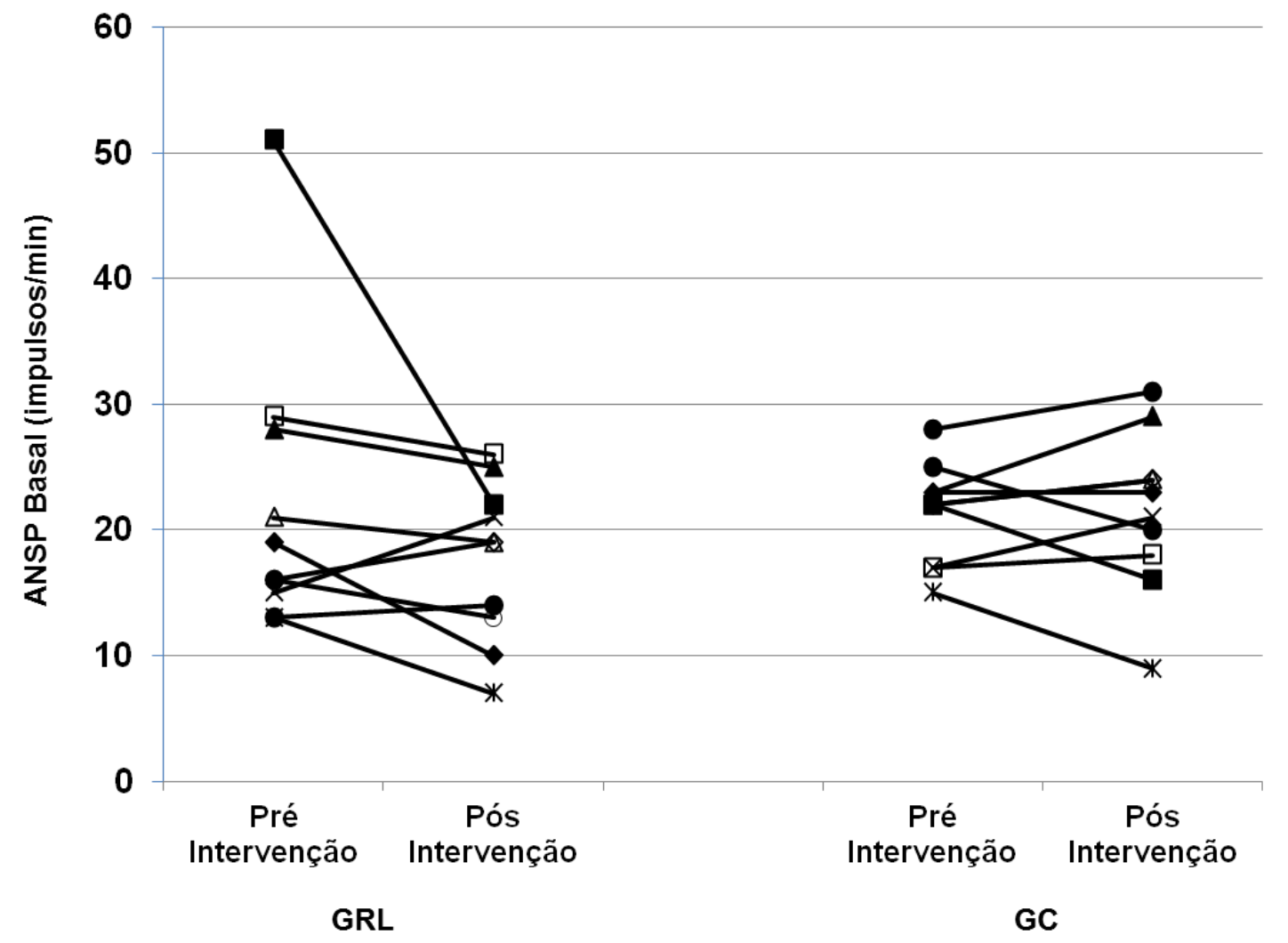

Figura 8 - Atividade nervosa simpática periférica (ANSP) medida pela microneurografia antes e após intervenção no Grupo Respiração Lenta (GRL) e Grupo Controle (GC) 
A Tabela 6 mostra o controle neurovascular frente às manobras de estimulação da atividade nervosa simpática. No GC observou-se uma tendência de atenuação do aumento da atividade nervosa simpática frente às manobras excitatórias, particularmente durante a fase de oclusão, entre os períodos pré-intervenção ( $\Delta 6 \pm 14$ impulsos/minuto) e pós-intervenção $(\Delta-2 \pm 5$ impulsos/minuto). No entanto, tal diferença não se mostrou estatisticamente significante $(p>0,05)$.

Tabela 6 - Atividade Nervosa Simpática Periférica (ANSP), medida pela microneurografia, antes e após a intervenção de acordo com a randomização, Grupo Respiração Lenta (GRL) versus Grupo Controle (GC)

\begin{tabular}{lccccccc}
\hline \multirow{2}{*}{ Variável } & \multicolumn{3}{c}{ GRL $(\mathbf{n}=10)$} & \multicolumn{3}{c}{ GC $(\mathbf{n}=10)$} \\
\cline { 2 - 7 } & Pré & Pós & $\mathbf{p}$ & Pré & Pós & $p$ \\
\hline$\Delta$ ANSP Handgrip (impulsos/min) & $3 \pm 7$ & $2 \pm 3$ & 0,52 & $2 \pm 6$ & $-1 \pm 5$ & 0,32 \\
$\Delta$ ANSP Oclusão (impulsos/min) & $2 \pm 6$ & $3 \pm 5$ & 0,56 & $6 \pm 14$ & $-2 \pm 5$ & 0,09 \\
$\Delta$ ANSP Recuperação (impulsos/min) & $1 \pm 5$ & $2 \pm 03$ & 0,42 & $3 \pm 5$ & $-1 \pm 6$ & 0,13 \\
\hline
\end{tabular}

p>0,05; ANSP pré versus ANSP pós, para o Grupo Respiração Lenta e Grupo Controle.

$\Delta$ diferença da atividade nervosa simpática (impulso/minuto) medida durante a manobra excitatória e o período basal.

\section{7- Adesão à intervenção}

A adesão ao exercício de respiração lenta guiada, avaliada por meio das variáveis armazenadas na memória do dispositivo eletrônico e descarregadas a cada 30 dias, pode ser visualizada na Tabela 7. 
Tabela 7 - Adesão ao exercício de respiração lenta guiada

\begin{tabular}{|c|c|c|}
\hline Desempenho estatístico do dispositivo de respiração guiada & 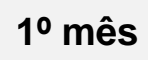 & $2^{\circ}$ mês \\
\hline Número de sessões realizadas & $28 \pm 5$ & $28 \pm 6$ \\
\hline $\begin{array}{l}\text { Média de tempo gasto (minutos) na zona de respiração } \\
\text { terapêutica (<10 respirações/minuto) }\end{array}$ & $13 \pm 4$ & $13 \pm 3$ \\
\hline Média da frequência respiratória inicial (rpm) & $10 \pm 3$ & $9 \pm 4$ \\
\hline Média da frequência respiratória final (rpm) & $6 \pm 2$ & $6 \pm 2$ \\
\hline $\begin{array}{l}\text { Média de tempo em que o sensor respiratório esteve apto a } \\
\text { identificar a respiração (\%) }\end{array}$ & $90 \pm 6$ & $91 \pm 4$ \\
\hline
\end{tabular}

rpm= respirações por minuto.

Como demonstrado na Tabela 7 os participantes do GRL tiveram boa adesão à intervenção, realizando o exercício proposto em média 28 dias dos 30 programados em cada mês e permaneceram 13 minutos na zona terapêutica (<10 respirações por minuto) dos 15 minutos recomendados. Além disso, a adesão à intervenção também foi avaliada pelo diário impresso, preenchido pelos participantes, que relataram que o dispositivo eletrônico foi utilizado em $93 \pm 3 \%$ do período proposto (60 dias), corroborando com os dados armazenados no dispositivo eletrônico.

Para o GC, a adesão ao tratamento somente foi avaliada pelo diário impresso preenchido pelos participantes, sendo relatado que o aparelho de MP3 foi utilizado em média $95 \pm 4 \%$ do período proposto (60 dias). 
5. Discussão 
O principal resultado do presente estudo foi evidenciar que a atividade nervosa simpática de hipertensos, com ou sem o uso de terapia antihipertensiva medicamentosa, medida tanto pela dosagem de catecolaminas plasmáticas quanto pela técnica da microneurografia, não é afetada pelo uso crônico do dispositivo de respiração lenta guiada (15 minutos diários durante 8 semanas). Além disso, nenhum efeito foi demonstrado na redução da PA de consultório e nas variáveis da MAPA de 24 horas.

Dois estudos prévios ${ }^{25,34}$ mostraram redução da ANSP após sessão isolada do exercício de respiração lenta guiada em pacientes hipertensos. No entanto, em um desses estudos ${ }^{34}$, que também avaliou o efeito do uso crônico (8 semanas) do exercício de respiração lenta guiada na ANSP, não demonstrou redução desta variável da mesma forma que a verificada na sessão aguda, corroborando, portanto, os dados do nosso estudo.

Uma possível explicação é a de que a redução da PA com o uso a longo prazo do dispositivo de respiração lenta guiada observada em alguns estudos não seja mediada pela redução da ANSP, ou seja, outros mecanismos estariam envolvidos. Contudo, tivemos a oportunidade de relatar um $\operatorname{caso}^{26}$ de redução expressiva da ANSP e das catecolaminas plasmáticas, após o uso crônico do dispositivo de respiração lenta guiada. Este caso, particularmente, chamou a atenção pela atividade nervosa simpática extremamente elevada no período basal (noradrenalina plasmática: 903 pg/ml; contagem de 51 impulsos nervosos por minuto pela microneurografia).

Podemos levantar a seguinte hipótese para explicar esta discrepância de resultados observados nestes diferentes estudos: o dispositivo eletrônico de respiração lenta guiada seria eficaz apenas em pacientes cujo mecanismo 
fisiopatológico responsável pela elevação da PA fosse predominantemente a hiperatividade simpática. O grupo de participantes incluídos no presente estudo tem características clínicas que não indicam atividade simpática exacerbada: hipertensos leves, não obesos, sem doença renal crônica e com noradrenalina plasmática basal dentro da normalidade.

Em uma subanálise dos dados do estudo, os participantes foram separados em dois grupos de acordo com a mediana da dosagem de noradrenalina no período basal com intuito de separar aqueles participantes com atividade nervosa simpática mais elevada.

Mesmo com esta divisão, os resultados foram semelhantes ao do grupo total, ou seja, os participantes com atividade nervosa simpática mais elevada não apresentaram redução da PA ou da concentração da noradrenalina plasmática após o período de intervenção. O que enfraquece esta observação é o número reduzido de indivíduos em cada grupo após a divisão (inferior a dez participantes).

Além disto, a esperada elevação da ANSP não foi atenuada durante as manobras excitatórias realizadas com aparelho de preensão manual (exercício isométrico) e com a oclusão do fluxo sanguíneo do antebraço no GRL. Tais achados são consistentes com o estudo previamente citado ${ }^{34}$ que mostrou que a respiração lenta guiada também não influenciou os efeitos cardiovasculares dos estressores físicos sobre a ANSP, embora tenha sido observada redução na ANSP quando medida em condições de estresse mental, parâmetro não avaliado no nosso estudo.

Resultado não esperado do estudo foi a elevação significativa da ANSP em relação ao período basal após as 8 semanas de intervenção para os dois grupos (GRL e GC). Do ponto de vista fisiológico, a redução da FR para algo 
em torno de 6-10 rpm, com a fase expiratória prolongada, aumenta o volume corrente e desencadearia um processo reflexo de redução da ANSP e consequente redução da $P A^{24}$. Neste estudo, o objetivo de redução da FR, por meio do dispositivo de respiração lenta guiada foi atingido, tendo em vista que os participantes finalizavam as sessões propostas com uma média de FR de $6 \pm 2$ rpm. Entretanto a redução da ANSP não foi alcançada nesta população de hipertensos conforme comentado anteriormente, e nem, tampouco, se observou a esperada redução da PA.

Em relação à $\mathrm{PA}$ de consultório e de 24 horas, nossos achados não demonstraram reduções significativas destas variáveis após o uso prolongado (8 semanas) do dispositivo de respiração lenta guiada. Esses achados corroboram os resultados de um estudo anterior ${ }^{19}$, realizado em uma população de hipertensos não diabéticos, que mostrou que o dispositivo de respiração lenta guiada não reduz a PAS e PAD de consultório e também a PA medida pela MAPA de 24 horas em maior magnitude que ouvir músicas serenas o fez (grupo controle). Outros dois estudos ${ }^{34,35}$, um com 8 semanas de utilização do dispositivo de respiração lenta guiada e o outro com 4 semanas, respectivamente, mostraram reduções na PA de consultório, porém, não houve efeitos nas médias de PA pela MAPA de 24 horas. Esse efeito de redução na PA de consultório, não reproduzido nos parâmetros da MAPA de 24 horas se deve, possivelmente, à modulação do efeito do avental branco ${ }^{34}$. No presente estudo, o efeito do avental branco foi excluído no momento da seleção dos voluntários, uma vez que todos os participantes incluídos no estudo foram escolhidos a partir de uma média de PA de 24 horas anormal pela MAPA, independente dos valores da PA de consultório. 
Apesar do exercício de respiração lenta guiada ser recomendado como uma estratégia eficaz na redução da pressão arterial pela American Heart Association $(\mathrm{AHA})^{21}$, os efeitos do dispositivo de respiração lenta guiada sobre a PA ainda são controversos. A maioria dos resultados publicados até 0 momento relatam efeitos favoráveis em relação à redução da pressão arterial de consultório de hipertensos sem outras comorbidades ${ }^{14,15,16,18,35}$ e hipertensos com $\mathrm{DM} 2^{17}$ com o uso do dispositivo de respiração lenta guiada, porém, há também, estudos relatando efeitos desfavoráveis relativos à redução da PA em hipertensos $\operatorname{com}^{20,36}$ e sem diabetes $^{19,34}$.

Em uma revisão sistemática ${ }^{37}$ e duas meta-análises ${ }^{38,39}$ recentemente publicadas, baseadas em estudos com qualidades metodológicas consideradas aceitáveis pelos autores, os resultados sugerem que o dispositivo de respiração lenta guiada é ineficaz em reduzir a PA de hipertensos quando utilizado por um período aproximado de 8 semanas. Portanto, o presente estudo se junta àqueles que não demonstraram redução da pressão arterial com o uso do aparelho de respiração lenta guiada, reforçando assim os resultados das meta-análises. Inclusive, há sugestão na literatura, de que esta recomendação da AHA seja revista ${ }^{40}$.

Uma observação que merece discussão foi a redução da PA em algumas variáveis da MAPA nos pacientes que escutaram música durante o período de intervenção do estudo.

A redução da $\mathrm{PA}$ em grupos controles de trabalhos envolvendo pacientes hipertensos não é novidade, seja usando medicamentos ${ }^{41}$, ou até mesmo quando da realização de terapias invasivas para controlar a $\mathrm{PA}^{42}$. O próprio efeito placebo poderia explicar estes achados. 
Outra explicação seria que o ato em si, de escutar música diariamente teria algum efeito hipotensor, não mediado pela redução da atividade nervosa simpática.

Particularmente no presente estudo, uma melhora na adesão à terapia anti-hipertensiva medicamentosa prescrita poderia explicar este achado. Apesar de termos dividido os participantes de forma aleatória, um maior percentual de participantes que faziam uso de anti-hipertensivos estava presente do subgrupo randomizado para ouvir música (75\% vs 23\%, $p<0,01)$. Apesar de não termos medido a adesão à terapia medicamentosa de forma objetiva, o fato de participar de um protocolo clínico de estudo pode ter melhorado a adesão à terapia medicamentosa e, consequentemente, levado a redução da pressão arterial observada.

Resultado que merece ser discutido foi a observação de elevação da frequência cardíaca nos participantes randomizados para o GRL após o período de intervenção, já que uma frequência cardíaca mais elevada poderia ser interpretada como um marcador de aumento da atividade nervosa simpática.

No entanto dois comentários sobre este resultado: a metodologia utilizada para a coleta deste dado foi pela análise dos resultados advindos da MAPA, metodologia padrão-ouro no que se refere ao estudo do comportamento da pressão arterial nas 24 horas, mas que necessita de validação quando se analisa a variável frequência cardíaca. O outro senão seria que apesar da elevação da frequência cardíaca ter sido significativa do ponto de vista estatístico, são praticamente desprezíveis do ponto de vista clínico, uma vez que foram de pequena magnitude (elevação abaixo de 5 bpm) e ainda dentro do intervalo considerado normal para a frequência cardíaca (entre 60 e 100 bpm). 
Portanto, o exercício de respiração lenta guiada, embora seja uma proposta interessante de tratamento não medicamentoso da hipertensão arterial, ainda necessita de maiores evidências da sua real efetividade. Caso este método venha se mostrar consistentemente capaz de reduzir a pressão arterial, os mecanismos responsáveis por esta redução devem ser mais amplamente estudados, não se atendo exclusivamente ao papel do sistema nervoso simpático. 
6. Conclusão 
O exercício de respiração lenta guiada realizado de maneira crônica (8 semanas) em hipertensos sem outras comorbidades não reduziu os valores da PA de consultório e as médias de PA de 24 horas. Também não foi observada diminuição da atividade nervosa simpática com o emprego desta técnica. 
Anexo A

Termo de Consentimento Livre e Esclarecido

\begin{abstract}
hOSPITAL DAS CLÍNICAS DA FACULdADE DE MEDICINA DA UNIVERSIDADE DE SÃO PAULO-HCFMUSP
\end{abstract}

TERMO DE CONSENTIMENTO LIVRE E ESCLARECIDO

DADOS DE IDENTIFICAÇÃO DO SUJEITO DA PESQUISA OU RESPONSÁVEL LEGAL

1. NOME:

DOCUMENTO DE IDENTIDADE №

SEXO :M $\square \quad F$

DATA NASCIMENTO:

ENDEREÇO: №

APTO:

BAIRRO:

CIDADE:

CEP:.

TELEFONE

$\mathrm{DDD}($

..)

2. RESPONSÁVEL LEGAL

NATUREZA (grau de parentesco, tutor, curador,etc.)

DOCUMENTO DE IDENTIDADE : SEXO: $M \square \quad F \square$

DATA NASCIMENTO.:

ENDEREÇO:

BAIRRO:.

$\mathrm{N}$

..APTO

CEP:

TELEFONE:

.CIDADE

\title{
DADOS SOBRE A PESQUISA
}

1. TítULO DO PROTOCOLO DE PESQUISA: Efeitos da respiração lenta na pressão arterial e na função autonômica em pacientes hipertensos.

PESQUISADOR PRINCIPAL: Dr. Décio Mion Jr.

CARGO/FUNÇÃO: Professor Livre Docente/Chefe da Unidade de Hipertensão do HCFMUSP INSCRIÇÃO CONSELHO REGIONAL № (CRM) 28828

PESQUISADOR EXECUTANTE: Silvana de Barros

CARGO/FUNÇÃO: Biologista do Laboratório de Hipertensão do HC-FMUSP INSCRIÇÃO ÇONSELHO REGIONAL № (CRBio) 51899/01-D.

UNIDADE DO HCFMUSP: Laboratório de Hipertensão do Serviço de Nefrologia

2. AVALIAÇÃO DO RISCO DA PESQUISA:

$\begin{array}{lll}\text { RISCO MÍNIMO } & \square & \text { RISCO MÉDIO } \\ \text { RISCO BAIXO } & X & \text { RISCO MAIOR }\end{array}$

3. DURAÇÃO DA PESQUISA : 18 meses 


\section{HOSPITAL DAS CLÍNICAS DA FACULDADE DE MEDICINA DA UNIVERSIDADE DE SÃO PAULO-HCFMUSP}

\section{DESENHO DO ESTUDO E OBJETIVOS:}

O senhor (a) está sendo convidado (a) a participar de uma pesquisa clínica que irá avaliar o comportamento da sua pressão arterial e do seu sistema nervoso ("nervos") antes e após o uso prolongado (08 semanas) de um aparelho que guiará sua respiração. O objetivo da pesquisa é mostrar se há ou não participação do seu sistema nervoso na redução da pressão arterial pelo uso do aparelho de respiração guiada.

\section{PROCEDIMENTOS QUE SERÃO REALIZADOS E PROPÓSITOS:}

Caso aceite participar do estudo, o senhor (a) realizará:

a) Monitorização Ambulatorial da Pressão arterial (MAPA): o senhor utilizará um aparelho automático e portátil para medir sua pressão arterial durante 24 horas. Com isso será possível avaliar sua pressão arterial durante o dia, enquanto realiza suas atividades diárias e durante a noite, enquanto dorme.

b) Visita ao Laboratório de Hipertensão onde serão realizadas:

- Medida do peso e altura

- Coleta de amostra de sangue para dosagem de hormônios.

- Medidas da pressão arterial que serão obtidas por dois diferentes aparelhos, sendo um aparelho semi-automático, como é habitual em hospitais e outro aparelho que irá medir a pressão arterial no dedo médio da sua mão.

- Eletrocardiograma para monitorar sua frequência cardíaca.

- Monitorização da respiração com o auxílio de uma cinta respiratória que será colocada sobre seu tórax.

- Microneurografia para verificar como seu sistema nervoso controla sua circulação sanguínea. Neste exame será colocado em sua perna, na lateral, logo abaixo do joelho, duas pequenas agulhas, bem finas, que fornecerão esse registro.

c) Utilização de um aparelho semelhante a um "Walkman" ou um aparelho de MP3 que irá auxiliar na redução da pressão arterial. O senhor (a) fará uso de um destes aparelhos por 15 minutos diários durante 8 semanas consecultivas.

\section{DESCONFORTOS E RISCOS ESPERADOS:}

Todos os exames desta pesquisa são seguros e bem tolerados. Porém a microneurografia poderá causar certos desconfortos durante sua realização, como por exemplo, leve dor no local onde serão colocadas as pequenas agulhas, formigamento e sensação de peso na perna e no pé. Esses desconfortos podem ou não aparecer durante o período de manipulação das agulhas, desaparecendo completamente após seu término.

\section{BENEFÍCIOS QUE PODERÃO SER OBTIDOS:}

Os benefícios do senhor (a) participar deste estudo são os de realizar uma avaliação detalhada da sua pressão arterial e do controle nervoso sobre seus vasos sanguíneos, bem como o uso de uma terapia não medicamentosa para auxiliar no controle e redução da pressão arterial, o que pode reduzir possíveis efeitos colaterais provenientes do aumento do uso de medicamentos ou doses prescritas. 
5. PROCEDIMENTOS ALTERNATIVOS QUE POSSAM SER VANTAJOSOS PARA O INDIVÍDUO:

Utilização de uma terapia não medicamentosa que auxilia na redução da pressão arterial sem que haja qualquer tipo de efeito colateral.

6. ACESSO, A QUALQUER TEMPO, ÀS INFORMAÇÕES SOBRE PROCEDIMENTOS, RISCOS E BENEFÍCIOS RELACIONADOS À PESQUISA, INCLUSIVE PARA DIMINUIR EVENTUAIS DÚVIDAS:

O senhor (a) tem o direito de acesso quando quiser a qualquer informação que deseje sobre a realização dos exames e procedimentos, bem como às informações contidas nesta declaração que ficará arquivada em seu prontuário. 0 principal investigador é o Dr. Décio Mion Jr. e o investigador executante a biologista Silvana de Barros, que podem ser encontrados na Avenida Dr. Enéas Carvalho de Aguiar, 255 - 7ํandar, sala 7031 Telefone 3069-7686. Se você tiver alguma consideração ou dúvida sobre a ética da pesquisa, entre em contato com o Comitê de Ética em Pesquisa (CEP) - Rua Ovídio Pires de Campos, 225 - 5o andar - tel: 3069-6442 ramais 16, 17, 18 ou 20, FAX: 3069-6442 ramal 26 - Email: cappesq@hcnet.usp.br

7. LIBERDADE DE RETIRAR SEU CONSENTIMENTO A QUALQUER MOMENTO E DE DEIXAR DE PARTICIPAR DO ESTUDO, SEM QUE ISTO TRAGA PREJUÍZO À CONTINUIDADE DA ASSISTÊNCIA:

A qualquer momento, independentemente do motivo e sem a necessidade de fornecer maiores explicações a estes pesquisadores, o senhor (a) poderá recusarse a continuar participando do estudo, sem que isto altere a continuidade de seu tratamento.

8. DIREITO DE CONFIDENCIALIDADE, SIGILO E PRIVACIDADE:

Apenas o senhor (a), os médicos e os membros do Comitê de Ética e autoridades regulatórias terão acesso às informações deste estudo. Os resultados dos seus exames serão analisados em conjunto com os outros pacientes, evitando mencionar seu nome ou seus dados pessoais sempre que possível, durante todo o estudo e também na publicação dos resultados.

9. DIREITO DE SER MANTIDO ATUALIZADO SOBRE OS RESULTADOS PARCIAIS DAS PESQUISAS:

O senhor (a) terá acesso a todos os resultados parciais dos exames realizados e, caso haja qualquer alteração, será encaminhado para o serviço responsável.

\section{DESPESAS E COMPENSAÇÕES:}

Não há despesas pessoais para o participante em qualquer fase do estudo, incluindo exames e consultas. Também não há compensação financeira relacionada à sua participação. Se existir qualquer despesa adicional, ela será absorvida pelo orçamento da pesquisa. 


\section{COMPROMISSO DO PESQUISADOR DE UTILIZAR OS DADOS E O MATERIAL COLETADO SOMENTE PARA ESTA PESQUISA:}

Os pesquisadores comprometem-se em utilizar os dados coletados durante este estudo (dados eletrônicos impressos, sua identificação, sua ficha clínica ou prontuário, os resultados de exames, etc.) somente para fins da pesquisa aqui proposta.

Acredito ter sido suficientemente informado a respeito das informações que li ou que foram lidas para mim, descrevendo o estudo "Avaliação da Pressão Arterial e da Função autonômica Pelo Uso da Respiração Guiada por Dispositivo Eletrônico em Pacientes Hipertensos".

Eu discuti com o Dr. Décio Mion Jr. (Pesquisador Responsável) e/ou com Silvana de Barros (Pesquisador Executante) sobre a minha decisão em participar neste estudo. Ficaram claros para mim quais são os propósitos do estudo, os procedimentos a serem realizados, seus desconfortos e riscos, as garantias de confidencialidade e de esclarecimentos permanentes. Ficou claro também que minha participação é isenta de despesas e que tenho garantia do acesso a tratamento hospitalar quando necessário. Concordo voluntariamente em participar deste estudo e poderei retirar o meu consentimento a qualquer momento, antes ou durante o mesmo, sem penalidades ou prejuízo ou perda de qualquer benefício que eu possa ter adquirido, ou no meu atendimento neste Serviço

Assinatura do paciente/representante legal Data

Assinatura da testemunha

Data_ $1 / 1$

para casos de pacientes menores de 18 anos, analfabetos, semi-analfabetos ou portadores de deficiência auditiva ou visual.

(Somente para o responsável do projeto)

Declaro que obtive de forma apropriada e voluntária o Consentimento Livre e Esclarecido deste paciente ou representante legal para a participação neste estudo. 


\title{
Anexo B
}

\section{Aprovação pela Comissão de Ética para Análise de Projetos de}

Pesquisa do Hospital das Clínicas da Faculdade de Medicina da

\author{
Universidade de São Paulo
}

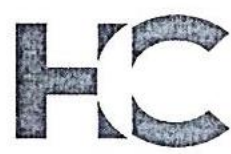

\section{APROVAÇÃO}

A Comissão de Ética para Análise de Projetos de Pesquisa CAPPesq da Diretoria Clínica do Hospital das Clínicas e da Faculdade de Medicina da Universidade de São Paulo, em sessão de 24/03/2010, APROVOU O Protocolo de Pesquisa $n^{\circ}$ 0107/10, intitulado: "EFEITOS DA RESPIRAÇÃO LENTA NA PRESSÃO ARTERIAL E NA FUNÇÃO AUTONÔMICA EM PACIENTES HIPERTENSOS" apresentado pelo Departamento de CLíNICA MÉDICA, inclusive o Termo de Consentimento Livre e Esclarecido.

Cabe ao pesquisador elaborar e apresentar à CAPPesq, os relatórios parciais e final sobre a pesquisa (Resolução do Conselho Nacional de Saúde n 196, de 10/10/1996, inciso IX.2, letra "c").

Pesquisador (a) Responsável: Prof. Dr. Décio Mion Júnior

Pesquisador (a) Executante: Silvana de Barros

Co-autor(es): Giovanio Vieira da Silva, Josiane Lima de Gusmāo, Crivaldo Gomes Cardoso Jr., Bruna Oneda, Katia Coelho Ortega

CAPPesq, 25 de Março de 2010

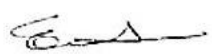

Prof. Dr. Eduardo Massad Presidente da Comissão de Ética para Análise de Projetos de Pesquisa

Comissão de Ética para Análise de Projetos de Pesquisa do HCFMUSP e da FMUSP Diretoria Clínica do Hospital das Clinicas da Faculdade de Medicina da Universidade de São Paulo Rua Ovídio Pires de Campos, 225, $5^{\circ}$ andar - CEP 05403010 - São Paulo - SP Fone: 01130696442 Fax: 01130696492 e-mail: cappesq@hcnet.usp.br/secretariacappesq2@hcnet.usp.br 
1. Chow CK, Teo KK, Rangarajan S, Islam S, Gupta R, Avezum A, et al. Prevalence, awareness, treatment, and control of hypertension in rural and urban communities in high-, middle-, and low-income countries. JAMA.2013; 310(9): 959-68.

2. Malachias MVB, Souza WKSB, Plavnik FL, Rodrigues CIS, Brandão AA, Neves MFT, et al. 7ํㅡㄹ Diretriz Brasileira de Hipertensão Arterial. Arq Bras Cardiol 2016; 107(3Supl.3):1-83.

3. Picon RV, Fuchs FD, Moreira LB, Riegel G, Fuchs SC. Trends in Prevalence of Hypertension in Brazil: A Systematic Review with MetaAnalysis. PLoS One. 2012; 7(10):e48255.

4. DATASUS - Tecnologia da Informação a Serviço do SUS. Disponível em <http://tabnet.datasus.gov.br/cgi/tabcgi.exe?sih/cnv/niuf.def>. Acesso em 11 Abril 2017.

5. Law MR, Morris JK, Wald NJ. Use of blood pressure lowering drugs in the prevention of cardiovascular disease: meta-analysis of 147 randomised trials in the context of expectations from prospective epidemiological studies. BMJ 2009; 338:b1665.

6. Mahmud A, Feely J. Low-Dose Quadruple Antihypertensive Combination More Efficacious Than Individual Agents - A Preliminary Report. Hypertension. 2007; 49: 272-5.

7. James PA, Oparil S, Carter BL, Cushman WC, Dennison-Himmelfarb C, Handler J, et al. 2014 evidence-based guideline for the management of high blood pressure in adults: report from the panel members appointed to the Eighth Joint National Committee (JNC 8). JAMA. 2014;311(5):507-20.

8. Mancia G, Fagard R, Narkiewicz K, Redón J, Zanchetti A, Böhm M, et al. $2013 \mathrm{ESH} / \mathrm{ESC}$ Guidelines for the management of arterial hypertension: the Task Force for the management of arterial hypertension of the European Society of Hypertension (ESH) and of the European Society of Cardiology (ESC). J Hypertens. 2013;31(7):1281-357.

9. Azadbakht L, Mirmiran P, Esmaillzadeh A, Azizi T, Azizi F. Beneficial Effects of a Dietary Approaches to Stop Hypertension Eating Plan on Features of the Metabolic Syndrome. Diabetes Care.2005; 28:2823-31.

10. Irvine MJ, Johnston DW, Jenner DA, Marie GV. Relaxation and Stress Management in the Treatment of Essencial Hypertension. J Psychosom Res.1986; 30: 437-50. 
11. McCaffrey R, Ruknui $P$, Hatthakit $U$, Kasetsomboon $P$. The Effects of Yoga on Hypertensive Persons in Thailand. Holist Nurs Pract.2005; 19(4): 173-80.

12. Chesney MA, Black GW, Swan GE, Ward MM. Relaxation Training for Essential Hypertension at the Worksite: I. The Untreated Mild Hypertensive. Psychosom Méd.1987; 49(3): 250-63.

13. Kaushik RM, Kaushik R, Mahajan SK, Rajesh V. Effects of Mental Relaxation and Slow Breathing in Essential Hypertension. Complement Ther Med. 2006; 14(2):120-6.

14. Schein MH, Gavish B, Herz M, Rosner-Kahana D, Naveh DP, Knishkowy B, et al.Treating Hypertension With a Device That Slows and Regularises Breathing: a Randomised, doble-blind Controlled Study. J Hum Hypertens.2001; 15: 271-8.

15. Grossman E, Grossman A, Schein MH, Zimlichman R, Gavish B. BreathingControl Lowers Blood Pressure. J Hum Hypertens.2001; 15: 263-9.

16. Elliot WJ, Izzo JL Jr, White WB, Rosing DR, Snyder CS, Alter A, et al. Graded blood pressure reduction in hypertensive outpatients associated with use of a device to assist with slow breathing. J Clin Hypertens. 2004; 6(10):553-9.

17. Schein MH, Gavish B, Baevsky T, Kaufman M, Levine S, Nessing A, et al. Treating hypertension in type II diabetic patients with device-guided breathing: a randomized controlled trial. J Hum Hypertens. 2009; 23(5):325-31.

18. Meles E, Giannattasio C, Failla M, Gentile G, Capra A, Mancia G. Nonpharmacologic Treatment of Hypertension by Respiratory Exercise in the Home Setting. Am J Hypertens. 2004; 17: 370-4.

19. Altena MR, Kleefstra N, Logtenberg SJ, Groenier KH, Houweling ST, Bilo HJ. Effect of device-guided breathing exercises on blood pressure in patients with hypertension: a randomized controlled trial. Blood Press. 2009;18(5):273-9.

20. Logtenberg SJ, Kleefstra N, Houweling ST, Groenier KH, Bilo HJ. Effect of device-guided breathing exercises on blood pressure in hypertensive patients with type 2 diabetes mellitus: a randomized controlled trial. $J$ Hypertens. 2007; 25(1):241-6. 
21. Brook RD, Appel LJ, Rubenfire M, Ogedegbe G, Bisognano JD, Elliott WJ, et al. American Heart Association Professional Education Committee of the Council for High Blood Pressure Research, Council on Cardiovascular and Stroke Nursing, Council on Epidemiology and Prevention, and Council on Nutrition, Physical Activity. Beyond medications and diet: alternative approaches to lowering blood pressure: a scientific statement from the American Heart Association. Hypertension. 2013;61:1360-1383.

22. Joseph CN, Porta C, Casucci G, Casiraghi N, Maffeis M, Rossi M, et al. Slow Breathing Improves Arterial Baroreflex Sensitivity and Decreases Blood Pressure in Essential Hypertension. Hypertension. 2005; 46: 714-8.

23. Radaelli A, Raco R, Perfetti P, Viola A, Azzellino A, Signorini MG, et al. Effects of Slow, Controlled Breathing on Baroreceptor Control of Heart Hate and Blood Pressure in Healthy Men. J Hypertens. 2004; 22:1361-70.

24. Parati G, et al. Respiration and Blood Pressure. In: Izzo Junior JL et al. Hypertension Primer: The Essentials of High Blood Pressure: Basic Science, Population Science, and Clinical Management. 4th ed. Dalas, Texas: American Heart Association, 2008. 136-8.

25. Oneda B, Ortega KC, Gusmão JL, Araújo TG, Mion D Jr. Sympathetic nerve activity is decreased during device-guided slow breathing. Hypertens Res. 2010 Jul;33(7):708-12.

26. de Barros S, da Silva GV, de Gusmão JL, de Araujo TG, Mion D Jr. Reduction of sympathetic nervous activity with device-guided breathing. $J$ Clin Hypertens. 2014;16(8):614-5.

27. V Diretrizes Brasileiras de Monitorização Ambulatorial da Pressão Arterial (MAPA V) e III Diretrizes Brasileiras de Monitorização Residencial da Pressão Arterial (MRPA III). Sociedades Brasileiras de Cardiologia, Hipertensão e Nefrologia. Arq Bras Cardiol 2011; 97(3 Supl 3):1-24.

28. Levey AS, Stevens LA, Schmid CH, Zhang YL, Castro AF 3rd, Feldman HI, et al. CKD-EPI (Chronic Kidney Disease Epidemiology Collaboration). A new equation to estimate glomerular filtration rate. Ann Intern Med. 2009;150(9):604-12. 
29. Jones CR, Taylor K, Chowienczyk P, Poston L, Shennan AH. A validation of the Mobil O Graph (version 12) ambulatory blood pressure monitor. Blood Press Monit. 2000;5(4):233-8.

30. Mano GM, Souza VF, Pierin AM, Lima JC, Ignes EC, Ortega KC, et al. Assessment of the DIXTAL DX-2710 automated oscillometric device for blood pressure measurement with the validation protocols of the British Hypertension Society (BHS) and the Association for the Advancement of Medical Instrumentation (AAMI). Arq Bras Cardiol. 2002; 79(6):606-10.

31. Hagbarth KE, Vallbo, AB. Pulse and Respiratory Grouping of Sympathetic Impulses in Human Muscle Nerves. Acta Physiol. Scand.1968; 74:96-108.

32. Mark AL. Microneurography: A Technique for Study of the Sympathetic Nervous System in Human Hypertension. J Cardiovasc Pharmacol.1987; 10(5):S56-9.

33. Venturelli M, Cè E, Limonta E, Bisconti AV, Devoto M, Rampichini S, et al. Central and peripheral responses to static and dynamic stretch of skeletal muscle: mechano- and metaboreflex implications. J Appl Physiol (1985). 2017 Jan 1;122(1):112-120.

34. Hering D, Kucharska W, Kara T, Somers VK, Parati G, Narkiewicz K. Effects of acute and long-term slow breathing exercise on muscle sympathetic nerve activity in untreated male patients with hypertension. $\mathrm{J}$ Hypertens. 2013 Apr;31(4):739-46.

35. Anderson DE, McNeely JD, Windham BG. Regular slow-breathing exercise effects on blood pressure and breathing patterns at rest. J Hum Hypertens. 2010;24(12):807-13.

36. Landman GW, Drion I, van Hateren KJ, van Dijk PR, Logtenberg SJ, Lambert $\mathrm{J}$, et al. Device-guided breathing as treatment for hypertension in type 2 diabetes mellitus: a randomized, double-blind, sham-controlled trial. JAMA Intern Med. 2013;173(14):1346-50. 
37. van Hateren KJ, Landman GW, Logtenberg SJ, Bilo HJ, Kleefstra N. Device-guided breathing exercises for the treatment of hypertension: An overview. World J Cardiol. 2014;6(5):277-82.

38. Mahtani KR, Nunan D, Heneghan CJ. Device-guided breathing exercises in the control of human blood pressure: systematic review and meta-analysis. J Hypertens. 2012;30(5):852-60.

39. Landman GW, van Hateren KJ, van Dijk PR, Logtenberg SJ, Houweling ST, Groenier $\mathrm{KH}$, et al. Efficacy of device-guided breathing for hypertension in blinded, randomized, active-controlled trials: a meta-analysis of individual patient data. JAMA Intern Med. 2014;174(11):1815-21.

40. van Dijk PR, Landman GW, van Hateren KJ, Logtenberg SJ, Bilo HJ, Kleefstra N. Call for a re-evaluation of the American Heart Association's standpoint concerning device-guided slow breathing using the RESPeRATE device. Hypertension. 2013;62(4):e17.

41. Beckett NS, Peters R, Fletcher AE, Staessen JA, Liu L, Dumitrascu D, et al. HYVET Study Group. Treatment of hypertension in patients 80 years of age or older. N Engl J Med. 2008;358(18):1887-98.

42. Bhatt DL, Kandzari DE, O'Neill WW, D'Agostino R, Flack JM, Katzen BT, et al. SYMPLICITY HTN-3 Investigators. A controlled trial of renal denervation for resistant hypertension. N Engl J Med. 2014;370(15):1393-401. 